The updated version of this paper is available at http://www.bostonfed.org/economic/wp/wp2011/wp1103.htm as Working Paper No. 11-3,

"Estimation of Forward-Looking Relationships in Closed Form: An Application to the New Keynesian Phillips Curve." 
No. 09-15

\title{
Closed-Form Estimates of the New Keynesian Phillips Curve with Time-Varying Trend Inflation
}

\section{Michelle L. Barnes, Fabià Gumbau-Brisa, Denny Lie, and Giovanni P. Olivei}

\begin{abstract}
:
We compare estimates of the New Keynesian Phillips Curve (NKPC) when the curve is specified in two different ways. In the standard difference equation (DE) form, current inflation is a function of past inflation, expected future inflation, and real marginal costs. The alternative closed form (CF) specification explicitly solves the DE form to express inflation as a function of past inflation and a present-discounted value of current and expected future marginal costs. The CF specification places model-consistent constraints on expected future inflation that are not imposed in the DE form. In a Monte Carlo exercise, we show that estimating the CF version of the NKPC gives estimates that are much more efficient than the estimates obtained from the DE specification. We then compare DE and CF estimates of the NKPC with time-varying trend inflation on actual data. The data and estimation methodology are the same as in Cogley and Sbordone (2008). We show that DE and CF estimates differ substantially and have very different implications for inflation dynamics. As in Cogley and Sbordone, it is possible to estimate DE specifications of the NKPC where lagged inflation plays no role once trend inflation is taken into account. The CF estimates of the NKPC, however, typically imply as large a role for lagged inflation as for expected future inflation. These estimates thus suggest that trend inflation is not in itself sufficient to explain the persistent dynamics of inflation.
\end{abstract}

\section{JEL Classifications: E12, E31, E52}

Michelle L. Barnes is a senior economist and policy advisor, Fabià Gumbau-Brisa is a senior economist, and Giovanni P. Olivei is a vice president and economist, all at the Federal Reserve Bank of Boston. Their e-mail addresses are, michelle.barnes@bos.frb.org, fabià.gumbau-brisa@bos.frb.org, and giovanni.olivei@bos.frb.org respectively. Denny Lie is a research associate at the Federal Reserve Bank of Boston and is affiliated with Boston University. His email address is dlie@bu.edu.

This paper, which may be revised, is available on the web site of the Federal Reserve Bank of Boston at http://www.bos.frb.org/economic/wp/index.htm.

The views and opinions expressed in this paper are those of the authors and do not necessarily represent the views of the Federal Reserve Bank of Boston or the Federal Reserve System.

This version: December 12, 2009. The June 2011 version of this paper is available as Working Paper No. 11-3 at http://www.bostonfed.org/economic/wp/wp2011/wp1103.htm 


\section{Introduction}

In this paper we illustrate the differences that arise from estimating a New Keynesian Phillips curve (NKPC) when the relationship is expressed in two different but related forms. The first form, which we call the "difference equation" (DE) specification, is the standard Euler equation formulation where inflation is a function of past inflation, expected next-period inflation, and the driving process for inflation. The second form, which we call the "closed form" (CF) specification, takes the DE form and solves out iteratively for inflation expectations to obtain an expression where inflation is a function of past inflation and the expected present discounted value of the driving process for inflation. The $\mathrm{CF}$ specification imposes model-consistent constraints on expectations which are not imposed in the DE form. In essence, the CF specification recognizes that inflation needs to satisfy the DE form at any point in time, and forces inflation expectations to behave accordingly. While the CF version of the NKPC implies the DE version, the opposite is not always true.

When estimating the DE form of the NKPC, next-period inflation is instrumented by means of some unconstrained reduced-form model for inflation. If this unconstrained reduced-form model were identical to the "true" data generating model, then the DE and the CF specifications would be equivalent. But it is fair to assume that any unconstrained reduced-form for the "true" model underlying actual data is bound to be, at best, an approximation. If this is the case, then the DE and $\mathrm{CF}$ specifications are not equivalent. Absent such equivalence, it becomes important to ask whether estimates of economically relevant structural parameters differ when the relationship at hand is expressed in closed form rather than in the difference equation form.

In this paper, we show that deep parameter estimates of the NKPC obtained from the DE and $\mathrm{CF}$ specifications can differ substantially. The $\mathrm{CF}$ estimates are much more precise and more robust to a particular form of misspecification. This is shown both in the context of a Monte Carlo exercise and on actual U.S. data. For the estimation on actual data, we use a NKPC with time-varying coefficients as in Cogley and Sbordone (2008). These authors estimate a DE form of the NKPC, and we show how the estimates change when we consider instead the CF specification. The empirical exercise is conducted using the same data and the same estimation methodology as in Cogley and Sbordone. The DE estimates imply that, once taking into account time-varying trend inflation, the NKPC is purely forward-looking. The corresponding CF estimates, however, find an important role for lagged inflation. Indeed, according to the CF estimates lagged and 
expected future inflation enter the Euler equation form of the NKPC with rather similar weights. Another important dimension in which the DE and CF estimates differ is the frequency with which prices are readjusted optimally. In the DE specification this frequency is estimated at 3.9 months, while in the CF specification it is close to one year. ${ }^{1}$ Overall, the CF estimates of the NKPC with time-varying trend inflation suggest that U.S. inflation has an important persistent component that cannot be accounted for entirely by time-varying trend inflation or by persistence in the inflation driving process.

There is now a large literature on estimating NKPC models. ${ }^{2}$ The forward-looking component in the NKPC is usually derived from a micro-founded problem in which firms cannot reset prices optimally in every period. Firms then take into account not only current market conditions, but also expected future conditions when setting prices optimally. This mechanism alone provides no role for lagged inflation in the NKPC. But in actual data, inflation can be highly persistent and purely forward-looking versions of the NKPC often fit the data worse than "hybrid" versions where current inflation depends not just also on expected next period inflation, but also on past inflation. The dependence on past inflation is frequently introduced through some ad-hoc pricing mechanism (for example, indexation or "rule-of-thumb" price setters). This is unsatisfactory for many purposes, as the mechanism lacks micro-foundations. The work by Cogley and Sbordone is innovative in the literature in that it explores the possibility that the persistence in the inflation process is due to a time-varying inflation target rather than to some ad-hoc element in firms' price setting decisions. ${ }^{3}$ There is considerable evidence that the Federal Reserve's inflation target has not remained constant over time (Ireland, 2007), and this raises the possibility that variations in the target are an important source of inflation persistence. The empirical findings in Cogley and Sbordone do indeed favor a purely forward-looking Phillips curve where inflation persistence results entirely from a time-varying inflation target. These findings, therefore, are consistent with a price-setting framework that does not have to rely on some form of ad-hoc backward-looking price

\footnotetext{
${ }^{1}$ Our CF estimates find an important role for indexation to past inflation. In the presence of indexation, firms change prices every period (some are re-optimizing, while others are not). Hence the frequency of price re-optimization in this case cannot be directly compared to micro-evidence based on price changes alone.

${ }^{2}$ See, among others, Galí and Gertler (1999), Galí, Gertler, and López-Salido (2005), Rudd and Whelan (2006), and Sbordone (2002).

${ }^{3}$ Kozicki and Tinsley (2002) is the first study to explicitly consider time-varying trend inflation when estimating a NKPC. Cogley Sbordone, however, provide a full derivation of the NKPC with time-varying inflation from the firms' optimization problem, and their empirical exercise is tightly linked to the theoretical model.
} 
adjustment. $^{4}$

The implications of Cogley and Sbordone's findings are very important when thinking about inflation dynamics. As long as the inflation target is not moving, inflation is purely forward-looking and as persistent as its driving process. Consider for example a situation in which real marginal costs drop below their steady-state level and are expected to revert to the steady state in one year. Then inflation drops immediately and returns to its target level in one year, in sync with real marginal costs. ${ }^{5}$ The same is true for a markup shock. A one-period markup shock, for example, has only a one-period effect on inflation. Instead, when inflation is not purely forward-looking, the adjustment of inflation to movements in real marginal costs or to markup shocks is slower. A one-period negative markup shock, for example, results in lower current inflation. Given the dependence of next-period inflation on current inflation, this in turn lowers inflation in the next period. Indeed, inflation converges only asymptotically to the target, despite the one-time shock. These differences in inflation dynamics can have substantial implications for the design of optimal monetary policy. ${ }^{6}$

While the focus of this paper is the NKPC model, our Monte Carlo results on the difference between DE and CF estimates generalize to other macro relationships that can be written in the form of an Euler equation. The Monte Carlo findings favor the CF specification over the DE specification in terms of precision of the estimates. Additionally, the CF estimates are less sensitive to a form of misspecification that appears to be relevant in Cogley and Sbordone's setup on actual U.S. data. Using their same data and estimation method, we replicate Cogley and Sbordone's benchmark findings based on a DE specification of the NKPC with time-varying trend inflation. This specification allows for one-lag inflation indexation. We then show that even maintaining the DE specification, some of the parameter estimates change substantially when allowing for two-lag inflation indexation. In particular, lagged inflation enters significantly in the NKPC. The dependence of current inflation on lagged inflation is even stronger and more precisely estimated when we use CF versions of the NKPC.

The estimation procedure we use in the paper involves two steps. The first step produces a forecasting rule for inflation and real marginal costs based on an unconstrained vector autoregression

\footnotetext{
${ }^{4}$ The usual ad-hoc assumption of Calvo (1983) pricing notwithstanding.

${ }^{5}$ The NKPC model in Cogley and Sbordone features terms other than real marginal costs as additional driving processes, but their role in explaining inflation dynamics is estimated to be very small.

${ }^{6}$ See, for example, Benigno and López-Salido (2006) and Steinsson (2003).
} 
(VAR). Given the estimated forecasting rule, the second step uses minimum-distance methods to estimate the deep parameters of the NKPC. The forecasting rule is the same when estimating the DE and CF specifications, and this allows to isolate the effects of imposing the additional modelconsistent constraints in the $\mathrm{CF}$ specification. In this respect, our work contributes to previous literature (Fuhrer, Moore, and Schuh, 1995, and Fuhrer and Olivei, 2005) that compared DE and CF relationships, albeit in different settings and using different estimation methods. In those papers, the $\mathrm{CF}$ relationship is estimated in a single stage by means of full-information methods, so that the forecasting rule differs from the reduced-form forecasting rule used to estimate the DE specification. Moreover, in this paper we provide a formal explanation for the difference in the DE and $\mathrm{CF}$ estimates. We also illustrate how to improve on the DE estimates by placing some of the model-consistent constraints without the need to resort to the closed-form model solution. This is particularly convenient when the closed form is difficult to compute and in instrumental variables estimation settings where the closed form involves infinite sums of present-discounted values which, at best, can only be approximated. In this regard, our paper links the estimation problem of Euler equations to the minimum-distance and GMM estimators literature on the gains in efficiency that result from imposing additional constraints (Kodde, Palm, and Pfann, 1990, and Hayashi, 2000, ch.3).

The rest of the paper proceeds as follows. In section 2 we describe the DE and CF relationships in the context of a simple NKPC model, and discuss the two-stage estimation procedure. We then provide an explanation for the gain in efficiency from estimating the CF relationship and provide some Monte Carlo evidence. In section 3 we consider a NKPC model that allows for time-varying trend inflation and compare DE and CF estimates based on actual U.S. data. Section 4 offers some concluding remarks.

\section{A Simple New Keynesian Phillips Curve Framework: Estima- tion Methodology and Monte Carlo Simulations}

In order to convey the main points of the paper, in this section we use a conventional fixedcoefficients setup for the New-Keynesian Phillips curve. This setup does not allow for a timevarying inflation target, and thus it is simpler than the time-varying coefficients version of the NKPC developed in Cogley and Sbordone. We do so for simplicity of exposition, as our main 
results do not hinge on these specifics. We consider the same NKPC specification as in Christiano, Eichenbaum, and Evans (2005). ${ }^{7}$ In this framework, firms that do not change optimally their price in a given period through the Calvo (1983) random drawing can still update their current price. The updating follows an indexation mechanism based on the previous period aggregate inflation rate, and the degree to which indexation occurs is governed by the parameter $\rho \in[0,1]$, with $\rho=0$ denoting absence of indexation (and thus no mechanical updating) and $\rho=1$ full indexation. The latter case yields a NKPC relationship that depends almost as much on expected future inflation as on lagged inflation. In this setup, the difference equation (DE) specification of the NKPC takes the following form ${ }^{8}$

$$
\pi_{t}=\rho \pi_{t-1}+\beta\left(E_{t} \pi_{t+1}-\rho \pi_{t}\right)+\zeta m c_{t}+u_{t} .
$$

In equation (1), $\pi$ denotes inflation and $m c$ real marginal costs, while $E_{t}$ is the expectations operator conditional on the available information at time $t$. The parameter $\beta$ is a discount factor, while $\zeta$ is a function of the model's structural parameters, with $\zeta=(1-\alpha)(1-\alpha \beta) /(\alpha+\alpha \theta \omega)$. In this expression, $(1-\alpha)$ denotes the firms' probability of adjusting prices optimally each period, $\theta$ is the elasticity of substitution among goods, and $\omega$ is the elasticity of firms' marginal costs to their own output (a measure of the degree of strategic complementarity in pricing decisions across firms). The unpredictable error term $u$ is assumed to be i.i.d., and can be thought of as capturing potential misspecifications in the relationship or shocks to firms' desired mark-up. Rearranging (1) gives the following expression for period $t$ inflation

$$
\pi_{t}=\frac{\rho}{1+\beta \rho} \pi_{t-1}+\frac{\beta}{1+\beta \rho} E_{t} \pi_{t+1}+\frac{\zeta}{1+\beta \rho} m c_{t}+\widetilde{u}_{t}
$$

From either (1) or (2), it is possible to obtain a closed-form representation of inflation conditional on the expected discounted path of real marginal costs. Since the relationship in (2) holds in every period, the one-period-ahead discounted inflation expectations can be written as

$$
\beta E_{t} \pi_{t+1}=\frac{\beta \rho}{1+\beta \rho} \pi_{t}+\frac{\beta^{2}}{1+\beta \rho} E_{t} \pi_{t+2}+\frac{\zeta \beta}{1+\beta \rho} E_{t} m c_{t+1}
$$

Similarly, the two-period-ahead discounted inflation expectations are

$$
\beta^{2} E_{t} \pi_{t+2}=\frac{\beta^{2} \rho}{1+\beta \rho} E_{t} \pi_{t+1}+\frac{\beta^{3}}{1+\beta \rho} E_{t} \pi_{t+3}+\frac{\zeta \beta^{2}}{1+\beta \rho} E_{t} m c_{t+2},
$$

\footnotetext{
${ }^{7}$ See also Sbordone (2002).

${ }^{8}$ See Woodford (2003) and Christiano, Eichenbaum, and Evans (2005) for a derivation.
} 
and so on. Substituting iteratively these expressions into (2) or, equivalently, summing the lefthand and the right-hand sides of these expressions from time $t$ onward, we obtain the closed-form $(\mathrm{CF})$ representation of the $\mathrm{NKPC}^{9}$

$$
\pi_{t}=\rho \pi_{t-1}+\zeta \sum_{i=0}^{\infty} \beta^{i} E_{t} m c_{t+i}+u_{t} .
$$

The difference between equations (1) and (4) is that the CF representation explicitly incorporates model-consistent expectations about future inflation, whereas in (1) expectations about future inflation - the second term on the right-hand side of equation (2) - are unconstrained linear projections. The main results in our paper hinge on the way inference about the NKPC's structural parameters changes when estimating the CF representation (4) instead of the DE specification (1).

\subsection{Estimating the NKPC Structural Parameters}

The ultimate goal of the estimation procedure is to provide inference about the NKPC structural parameters $\alpha, \beta, \theta, \rho$, and $\omega$ (or a subset of these parameters), which we collect in the vector $\boldsymbol{\psi}=[\alpha, \beta, \theta, \rho, \omega]^{\prime}$. The procedure in Cogley and Sbordone exploits cross-equations restrictions between the NKPC structural parameters and the parameters of a reduced-form VAR. Consider a (column) vector of variables $\mathbf{x}$ that includes, possibly among others, inflation and real marginal costs. We assume that the law of motion for $\mathbf{x}$ can be represented by a reduced-form VAR of order $p$. Defining the vector $\mathbf{z}_{t}=\left(\mathbf{x}_{t}^{\prime}, \mathbf{x}_{t-1}^{\prime}, \ldots, \mathbf{x}_{t-p+1}^{\prime}\right)^{\prime}$, it is possible to rewrite the $\operatorname{VAR}(p)$ in first-order form as

$$
\mathbf{z}_{t}=\mathbf{A} \mathbf{z}_{t-1}+\varepsilon_{z, t}
$$

where $\mathbf{A}$ is a square matrix of coefficients. ${ }^{10}$ For simplicity and without loss of generality, we are omitting constants. ${ }^{11}$ In what follows, we assume that the solution to the NKPC model for the variables in $\mathbf{x}$ has a reduced-form representation that is captured by (5). This relationship is then used to form expectations about the variables of interest, inflation and real marginal costs. We can

\footnotetext{
${ }^{9}$ The closed-form representation of inflation can also be obtained from equation (1) by forward iteration of $E_{t}\left(\pi_{t+j}-\rho \pi_{t+j-1}\right), j \geq 1$.

${ }^{10}$ If $\mathbf{x}_{t}$ contains $n$ variables, then $\mathbf{z}_{t}$ is a vector of size $n \cdot p$. Hence the matrix $\mathbf{A}$ is $(n \cdot p) \times(n \cdot p)$, with the VAR coefficients in the first $n$ rows. The matrix $\mathbf{A}$ has all roots inside the unit circle.

${ }^{11}$ The intercepts play a central role in the NKPC with time-varying trend inflation considered in section 3. In the present setup, they are immaterial.
} 
express the conditional expectation of a variable $y_{t+k} \in \mathbf{x}_{t+k}$ at time $t-1$ as

$$
E_{t-1} y_{t+k}=\mathbf{e}_{y}^{\prime} \mathbf{A}^{k+1} \mathbf{z}_{t-1}
$$

where the vector $\mathbf{e}_{y}^{\prime}$ selects variable $y_{t-1}$ in $\mathbf{z}_{t-1}$. Consider then taking expectations as of $t-1$ of the NKPC written in the DE form (2) using the forecasting rule (6). We have

$$
\mathbf{e}_{\pi}^{\prime} \mathbf{A} \mathbf{z}_{t-1}=\frac{\rho}{1+\beta \rho} \mathbf{e}_{\pi}^{\prime} \mathbf{I} \mathbf{z}_{t-1}+\frac{\beta}{1+\beta \rho} \mathbf{e}_{\pi}^{\prime} \mathbf{A}^{2} \mathbf{z}_{t-1}+\frac{\zeta}{1+\beta \rho} \mathbf{e}_{m c}^{\prime} \mathbf{A} \mathbf{z}_{t-1},
$$

with I denoting an identity matrix that conforms with $\mathbf{A}$. The left-hand side of (7) is the expectation of inflation from the reduced-form VAR. The right-hand side is the expectation of inflation based on the NKPC model. Equation (7) says that if the NKPC in (1) is the true data generating process for inflation, the reduced-form forecast and the NKPC-based forecast for inflation must be the same. Imposing that (7) holds for all realizations of $\mathbf{z}$, equation (7) can be rearranged and simplified to obtain a vector of non-linear restrictions involving the VAR coefficients matrix $\mathbf{A}$ and the NKPC structural parameters $\psi$ :

$$
\mathbf{e}_{\pi}^{\prime} \mathbf{A}=-\beta \rho \mathbf{e}_{\pi}^{\prime} \mathbf{A}+\rho \mathbf{e}_{\pi}^{\prime} \mathbf{I}+\beta \mathbf{e}_{\pi}^{\prime} \mathbf{A}^{2}+\zeta \mathbf{e}_{m c}^{\prime} \mathbf{A} \equiv \mathbf{g}^{D}(\mathbf{A}, \boldsymbol{\psi}),
$$

or

$$
\mathbf{F}^{D}(\mathbf{A}, \boldsymbol{\psi}) \equiv \mathbf{e}_{\pi}^{\prime} \mathbf{A}-\mathbf{g}^{D}(\mathbf{A}, \boldsymbol{\psi})=\underline{0}^{\prime}
$$

where $\underline{0}^{\prime}$ is a column vector of zeros with same size as $\mathbf{e}_{\pi}$, and the superscript $D$ indicates that the expressions correspond to the DE specification.

The estimation procedure in Cogley and Sbordone involves two steps. The first step consists of estimating the law of motion for $\mathbf{x}$ from an unrestricted reduced-form VAR as in (5). This yields an estimated coefficients matrix $\widehat{\mathbf{A}}$. Given this estimate, the second step involves searching for values of the NKPC structural parameters $\boldsymbol{\psi}$ that minimize the squared deviation of $\mathbf{g}^{D}(\widehat{\mathbf{A}}, \boldsymbol{\psi})$ from $\mathbf{e}_{\pi}^{\prime} \widehat{\mathbf{A}}$, that is

$$
\widehat{\boldsymbol{\psi}}^{D} \equiv \arg \min \mathbf{F}^{D}(\widehat{\mathbf{A}}, \boldsymbol{\psi}) \cdot \mathbf{F}^{D}(\widehat{\mathbf{A}}, \boldsymbol{\psi})^{\prime}
$$

So far, we have illustrated the estimation procedure using the NKPC written in the DE form (2). The same reasoning applies to the NKPC written in closed form, equation (4). In this case, time $t-1$ expectations of the NKPC conditional on the forecasting rule (6) are

$$
\mathbf{e}_{\pi}^{\prime} \mathbf{A} \mathbf{z}_{t-1}=\rho \mathbf{e}_{\pi}^{\prime} \mathbf{I} \mathbf{z}_{t-1}+\zeta \mathbf{e}_{m c}^{\prime}(\mathbf{I}-\beta \mathbf{A})^{-1} \mathbf{A} \mathbf{z}_{t-1}
$$


and the vector of non-linear restrictions involving the VAR coefficients matrix $\mathbf{A}$ and the NKPC structural parameters $\boldsymbol{\psi}$ takes the form

$$
\mathbf{e}_{\pi}^{\prime} \mathbf{A}=\rho \mathbf{e}_{\pi}^{\prime} \mathbf{I}+\zeta \mathbf{e}_{m c}^{\prime}(\mathbf{I}-\beta \mathbf{A})^{-1} \mathbf{A} \equiv \mathbf{g}^{C}(\mathbf{A}, \psi)
$$

or

$$
\mathbf{F}^{C}(\mathbf{A}, \boldsymbol{\psi})=\mathbf{e}_{\pi}^{\prime} \mathbf{A}-\mathbf{g}^{C}(\mathbf{A}, \psi)=\underline{0}^{\prime}
$$

where the superscript $C$ indicates that the expressions correspond to the $\mathrm{CF}$ specification. The first step of the estimation procedure remains the same as before, while the second step involves searching for values of the NKPC structural parameters $\boldsymbol{\psi}$ that minimize the squared deviation of $\mathbf{g}^{C}(\widehat{\mathbf{A}}, \boldsymbol{\psi})$ from $\mathbf{e}_{\pi}^{\prime} \widehat{\mathbf{A}}$, that is

$$
\widehat{\boldsymbol{\psi}}^{C}=\arg \min \mathbf{F}^{C}(\widehat{\mathbf{A}}, \boldsymbol{\psi}) \cdot \mathbf{F}^{C}(\widehat{\mathbf{A}}, \boldsymbol{\psi})^{\prime}
$$

To summarize, the minimum-distance problems in (10) and (14) are both based on a system of implicit equations $\mathbf{F}^{i}(\mathbf{A}, \boldsymbol{\psi})=\underline{0}^{\prime}$, for $i \in\{D, C\}$. The system of equations has $k$ equations and $l$ unknowns, where $l$ is equal to the size of the structural parameter vector $\boldsymbol{\psi}$, and $k$ is the size of the square VAR matrix A. ${ }^{12}$ This system of equations provides the basis for the estimation method. Hence, when we replace $\mathbf{A}$ with its approximation $\widehat{\mathbf{A}}$ in (10) and (14), the goal is to choose the estimate of $\boldsymbol{\psi}$ that makes the vector $\mathbf{F}^{i}(\widehat{\mathbf{A}}, \boldsymbol{\psi})$ as close as possible to zero. The minimization problems in (10) and (14) differ, even if the NKPC is the "true" data generating process for inflation and $\widehat{\mathbf{A}}$ is the same in both minimizations. The reason for this difference is that $\mathbf{A}$ is estimated with sampling error. Then, as long as the system of equations is over-identified $(k>l)$, the estimates $\widehat{\boldsymbol{\psi}}^{D}$ and $\widehat{\boldsymbol{\psi}}^{C}$ are also going to be different. It is only in the special case of exact identification $(k=l)$ that $\widehat{\boldsymbol{\psi}}^{D}=\widehat{\boldsymbol{\psi}}^{C}$. With an over-identified system, $\mathbf{g}^{D}(\mathbf{A}, \boldsymbol{\psi})$ equals $\mathbf{g}^{C}(\mathbf{A}, \boldsymbol{\psi})$ only when $\mathbf{A}$ is known. In this case, there exists a $\boldsymbol{\psi}$ such that (8) and (12) hold exactly. Then it does not matter which specification (DE or CF) of the NKPC is being estimated, since inflation forecasts generated from the reduced-form VAR with the true matrix $\mathbf{A}$ - the second element on the right-hand side of (7) - are perfectly model-consistent.

To see how the CF specification imposes model-consistent constraints on expectations that are

\footnotetext{
${ }^{12}$ The number of equations in the VAR is given by $n$, and $p$ is the order of the VAR. Then $k=n \cdot p$.
} 
not imposed on the DE form, note that it is possible to write $\mathbf{g}^{D}(\widehat{\mathbf{A}}, \boldsymbol{\psi})$ as follows

$$
\begin{aligned}
\mathbf{g}^{D}(\widehat{\mathbf{A}}, \psi) & \equiv-\beta \rho \mathbf{e}_{\pi}^{\prime} \widehat{\mathbf{A}}+\rho \mathbf{e}_{\pi}^{\prime} \mathbf{I}+\beta \mathbf{e}_{\pi}^{\prime} \widehat{\mathbf{A}}^{2}+\zeta \mathbf{e}_{m c}^{\prime} \widehat{\mathbf{A}} \\
& =\rho \mathbf{e}_{\pi}^{\prime} \mathbf{I}+\zeta \mathbf{e}_{m c}^{\prime}(\mathbf{I}-\beta \widehat{\mathbf{A}})^{-1} \widehat{\mathbf{A}}+\beta \mathbf{k}(\widehat{\mathbf{A}}, \boldsymbol{\psi}) \widehat{\mathbf{A}} \\
& =\mathbf{g}^{C}(\widehat{\mathbf{A}}, \boldsymbol{\psi})+\beta \mathbf{k}(\widehat{\mathbf{A}}, \boldsymbol{\psi}) \widehat{\mathbf{A}}
\end{aligned}
$$

where

$$
\mathbf{k}(\widehat{\mathbf{A}}, \psi)=\mathbf{e}_{\pi}^{\prime} \widehat{\mathbf{A}}-\rho \mathbf{e}_{\pi}^{\prime} \mathbf{I}-\zeta \mathbf{e}_{m c}^{\prime}(\mathbf{I}-\beta \widehat{\mathbf{A}})^{-1} \widehat{\mathbf{A}}
$$

It is then apparent that for $\mathbf{g}^{D}(\widehat{\mathbf{A}}, \boldsymbol{\psi})$ to equal $\mathbf{g}^{C}(\widehat{\mathbf{A}}, \boldsymbol{\psi})$, the restrictions $\mathbf{k}(\widehat{\mathbf{A}}, \boldsymbol{\psi})=\underline{0}^{\prime}$ must be imposed. These restrictions are model-consistent, and represent all of the constraints on inflation expectations' formation implied by the NKPC at any point in time. They need to be satisfied in order to obtain the closed-form of the NKPC, but they are not exploited in the difference equation form from which $\mathbf{g}^{D}(\widehat{\mathbf{A}}, \boldsymbol{\psi})$ derives. In this case, expectations about future inflation - the second element on the right-hand side of (7) - are mechanically formed with the estimated $\widehat{\mathbf{A}}$. Hence, they do not take explicitly into account that the behavior of future inflation is, too, constrained to follow the NKPC relationship. The absence of such a constraint is inconsequential only when the true matrix $\mathbf{A}$ in the forecasting rule (6) is known, as in this case the mechanical projections coincide with the model-consistent inflation forecasts.

The question of interest, therefore, is how inference about $\boldsymbol{\psi}$, given the estimated $\widehat{\mathbf{A}}$, changes when in the second stage of the estimation process we use the closed form instead of the difference equation version of the NKPC. Before taking our estimation to actual data, we address this issue first from an analytical standpoint and provide Monte Carlo results.

\subsection{The efficiency gains from imposing model-consistent constraints on inflation expectations}

In this subsection we illustrate the effect of placing model-consistent constraints on inflation expectations when estimating the DE specification of the NKPC. Suppose that we are interested in estimating the NKPC in DE form, but we require that the same equation be valid for at least two consecutive periods. The two equations involved in the estimation, therefore, are the following

$$
\begin{aligned}
E_{t-1} \pi_{t} & =E_{t-1}\left\{\rho \pi_{t-1}+\beta\left(\pi_{t+1}-\rho \pi_{t}\right)+\zeta m c_{t}\right\} \\
E_{t-1} \pi_{t+1} & =E_{t-1}\left\{\rho \pi_{t}+\beta\left(\pi_{t+2}-\rho \pi_{t+1}\right)+\zeta m c_{t+1}\right\} .
\end{aligned}
$$


These two equations can be translated into two sets of cross-equation restrictions, with each set containing $k$ restrictions: ${ }^{13}$

$$
\begin{aligned}
\mathbf{c}_{0}(\mathbf{A}, \boldsymbol{\psi}) & \equiv \mathbf{F}^{D}(\mathbf{A}, \boldsymbol{\psi})=\underline{0}^{\prime} \\
\mathbf{c}_{1}(\mathbf{A}, \boldsymbol{\psi}) & \equiv \mathbf{e}_{\pi}^{\prime} \mathbf{A}^{2}-\rho \mathbf{e}_{\pi}^{\prime} \mathbf{A}+\beta \rho \mathbf{e}_{\pi}^{\prime} \mathbf{A}^{2}-\eta \mathbf{e}_{M C}^{\prime} \mathbf{A}^{2}-\beta \mathbf{e}_{\pi}^{\prime} \mathbf{A}^{3}=\underline{0}^{\prime}
\end{aligned}
$$

Given the definitions in (8) and (9), it is immediate to show that

$$
\mathbf{c}_{1}(A, \boldsymbol{\psi})=\mathbf{c}_{0}(A, \boldsymbol{\psi}) \cdot \mathbf{A}
$$

In addition, since the square matrix $\mathbf{A}$ is full rank, equation (19) implies that the following must be true

$$
\mathbf{c}_{1}(\mathbf{A}, \boldsymbol{\psi})=\underline{0}^{\prime} \Leftrightarrow \mathbf{c}_{0}(\mathbf{A}, \boldsymbol{\psi})=\underline{0}^{\prime} .
$$

Because of (19) and (20), we do not need to estimate the model parameters taking into account all of the $2 \cdot k$ cross-equation conditions in (17) and (18). Instead, the $2 \cdot k$ conditions are equivalent to the following $k$ constraints

$$
\begin{aligned}
\mathbf{c}_{1}^{*}(\mathbf{A}, \boldsymbol{\psi}) & \equiv \mathbf{c}_{0}(\mathbf{A}, \boldsymbol{\psi})-\beta \mathbf{c}_{1}(\mathbf{A}, \boldsymbol{\psi})=\underline{0}^{\prime} \\
& =\mathbf{F}^{D}(\mathbf{A}, \boldsymbol{\psi}) \cdot(\mathbf{I}+\beta \mathbf{A})=\underline{0}^{\prime} .
\end{aligned}
$$

The minimum-distance estimation of $\boldsymbol{\psi}$ in this case yields estimates

$$
\widehat{\boldsymbol{\psi}}^{D+1} \equiv \arg \min \mathbf{F}^{D}(\widehat{\mathbf{A}}, \boldsymbol{\psi}) \cdot(\mathbf{I}+\beta \widehat{\mathbf{A}}) \cdot(\mathbf{I}+\beta \widehat{\mathbf{A}})^{\prime} \cdot \mathbf{F}^{D}(\widehat{\mathbf{A}}, \boldsymbol{\psi})^{\prime}
$$

where the superscript $D+j$ indicates that we are imposing that the DE formulation of the NKPC holds for $j$ consecutive additional periods. The use of $(\mathbf{I}+\beta \widehat{\mathbf{A}})$ in $(22)$ forces the minimumdistance estimation to penalize specific errors and correlations among the errors in $\mathbf{F}^{D}(\widehat{\mathbf{A}}, \boldsymbol{\psi})$. The minimization problem in (22), imposes explicitly the model discipline on inflation expectations by acknowledging that the difference equation NKPC (1) should also apply at $t+1$. In this way, the estimation takes into account that specific violations of the cross-equation restrictions $\mathbf{F}^{D}(\mathbf{A}, \boldsymbol{\psi})=$ $\underline{0}^{\prime}$ have greater consequences for the behavior of inflation than others. The DE problem in (10), instead, disregards the interactions among these errors. In essence, the DE specification (1) is

\footnotetext{
${ }^{13}$ Recall that $\mathbf{A}$ is a full-rank square matrix of size $k$.
} 
more agnostic about the way in which inflation expectations are formed, and attaches the same importance to all cross-equation restrictions in $\mathbf{F}^{D}(\mathbf{A}, \boldsymbol{\psi})=\underline{0}^{\prime}$.

Of course, we may want to discipline expectations not only one period ahead, but also two periods ahead. Following the same logic that resulted in (21), the sets of cross-equation restrictions involved in this problem (a total of $3 \cdot k$ constraints) are now equivalent to the $k$ constraints

$$
\mathbf{c}_{2}^{*}(\mathbf{A}, \boldsymbol{\psi}) \equiv \mathbf{F}^{D}(\mathbf{A}, \boldsymbol{\psi}) \cdot\left(\mathbf{I}+\beta \mathbf{A}+\beta^{2} \mathbf{A}^{2}\right)=\underline{0}^{\prime} .
$$

More generally, we may want to require model-consistent expectations for up to $j$ periods ahead in time. By induction, the set of $k$ cross-equation restrictions is then given by

$$
\mathbf{c}_{j}^{*}(\mathbf{A}, \boldsymbol{\psi}) \equiv \mathbf{F}^{D}(\mathbf{A}, \boldsymbol{\psi}) \cdot\left(\mathbf{I}+\ldots+(\beta \mathbf{A})^{j}\right)=\underline{0}^{\prime} .
$$

It then follows that imposing model discipline on inflation expectations at any future point in time, the infinite number of model-consistent constraints on expectations is equivalent to the following $k$ restrictions

$$
\begin{aligned}
\mathbf{c}_{\infty}^{*}(\mathbf{A}, \boldsymbol{\psi}) & \equiv \lim _{j \rightarrow \infty} \mathbf{F}^{D}(\mathbf{A}, \boldsymbol{\psi}) \cdot\left(\mathbf{I}+\ldots+(\beta \mathbf{A})^{j}\right) \\
& =\mathbf{F}^{D}(\mathbf{A}, \boldsymbol{\psi}) \cdot(\mathbf{I}-\beta \mathbf{A})^{-1}=\underline{0}^{\prime}
\end{aligned}
$$

Combining the definitions (8), (9), (12), and (13), it is immediate to show that the DE and CF cross-equation restrictions are related by

$$
\mathbf{F}^{D}(\mathbf{A}, \boldsymbol{\psi}) \cdot(\mathbf{I}-\beta \mathbf{A})^{-1}=\mathbf{F}^{C}(\mathbf{A}, \boldsymbol{\psi})
$$

The $k$ cross-equation restrictions $\mathbf{c}_{\infty}^{*}(\mathbf{A}, \psi)=\underline{0}^{\prime}$ are then the very same restrictions that need to hold for the closed form NKPC:

$$
\mathbf{c}_{\infty}^{*}(\mathbf{A}, \boldsymbol{\psi})=\mathbf{F}^{C}(\mathbf{A}, \boldsymbol{\psi})=\underline{0}^{\prime} .
$$

Hence, as long as the NKPC model provides a good characterization of the data for every $j$ consecutive periods, the $k$ cross-equation restrictions in $\mathbf{c}_{j}^{*}(\mathbf{A}, \boldsymbol{\psi})$ provide more information about the model dynamics than the $k$ restrictions in $F^{D}(\mathbf{A}, \psi)$ derived from the DE problem alone. This additional information is the source of the gains in efficiency from estimating the CF specification versus the DE specification. The discussion so far also indicates that whenever the closed form is too complex to solve or too difficult to approximate reasonably well, it is possible to improve on the 
DE estimates by imposing additional constraints. These take the form of the DE relationship being iterated forward for $j \geq 1$ periods, possibly a much easier task than computing the closed form. We have shown that this is equivalent to imposing the $k$ cross-equation restrictions $\mathbf{c}_{j}^{*}(\mathbf{A}, \boldsymbol{\psi})=\underline{\mathbf{0}}^{\prime}$. In sum, the results in this section are related to the literature that illustrates the gains in estimation efficiency from imposing additional restrictions (see Gouriéroux, Monfort and Trognon, 1985, and Kodde, Palm, and Pfann, 1990). In our context, we show that this gain in efficiency can be obtained by imposing additional model-consistent restrictions on inflation expectations. What is particular about our setup is that these additional constraints can always be collapsed into $k$ crossequations restrictions, no matter how many these constraints are. Indeed, we show that imposing an infinite number of restrictions on the future expected dynamics of inflation results in the same set of $k$ cross-equation restrictions exploited in the estimation of the closed-form version of the NKPC.

\subsection{Monte Carlo Simulations}

We now turn to analyze the properties of the estimated vectors of parameters $\widehat{\boldsymbol{\psi}}^{D}$ and $\widehat{\boldsymbol{\psi}}^{C}$ obtained from the minimization problems in (10) and in (14), respectively, in the context of a Monte Carlo exercise. We are interested in ascertaining whether the use of the closed form instead of the difference equation version of the NKPC yields to estimates of the vector of parameters $\psi$ that, in small samples, are noticeably different in terms of biasedness and efficiency. We consider first a case in which the NKPC being estimated is the true data generating process, so that there are no misspecification issues. We then consider one case of misspecification that we deem relevant in actual data. Specifically, we generate data from a NKPC where lagged inflation enters as a weighted average of $t-1$ and $t-2$ inflation through the indexation mechanism, but then estimate a NKPC specification that only allows for lagged inflation at time $t-1$.

\subsubsection{No misspecification}

The artificial data for inflation in the Monte Carlo exercise are generated according to the NKPC (1). For the marginal costs process, we use a simple univariate $\mathrm{AR}(2)$ law of motion. For each of the Monte Carlo repetitions, we estimate a reduced-form VAR with two lags in inflation and marginal costs. The VAR estimation is carried out equation-by-equation via OLS. This provides us with an estimated matrix $\widehat{\mathbf{A}}_{(s)}$, where $s$ denotes the $s$-th repetition of the Monte Carlo experiment. 
With this reduced-form coefficients matrix, we can then estimate $\widehat{\boldsymbol{\psi}}_{(s)}^{D}$ using (10), and $\widehat{\boldsymbol{\psi}}_{(s)}^{C}$ using (14). ${ }^{14}$

Several considerations about this Monte Carlo exercise are in order. First, note that the NKPC we are estimating, whether in the $\mathrm{CF}$ or in the $\mathrm{DE}$ representation, is the true data generating process for inflation. In other words, there are no misspecification issues in this exercise. Second, the reduced-form process for real marginal costs, a univariate $\operatorname{AR}(2)$, is stylized but not overly counterfactual. The improvement in adjusted $R^{2}$ moving from the univariate $\operatorname{AR}(2)$ representation of marginal costs to a multivariate reduced-form representation that, in addition to two lags of real marginal costs, also includes two lags of inflation, the federal funds rate, and GDP growth, is only 1 percent in U.S. data over the period 1961:Q1 to 2003:Q4. ${ }^{15}$ The difficulty in working with this larger information set is that, in order to generate simulated inflation data, the NKPC in (1) needs to be solved first using standard rational expectations solution methods. The solution entails a constrained reduced-form representation of inflation that depends on $\psi$ and on the parameters describing the unconstrained reduced-form dynamics of the other variables. When using an augmented information set which, in addition to inflation and marginal costs, also includes the Federal funds rate and GDP growth, ${ }^{16}$ it is not possible to obtain unique or stable solutions for a range of relevant values of $\psi$. For this reason, we first report results from Monte Carlo simulations where the data generating process for real marginal costs is a simple univariate $\operatorname{AR}(2)$. In this case, it is possible to find a stable and unique solution for the NKPC model under a wide range of values for the vector $\psi$. We later show that our results do not change when the reduced-form dynamics of real marginal costs rely on a larger set of variables.

The $\operatorname{AR}(2)$ specification we use to generate the artificial data for marginal costs is the following

$$
m c_{t}^{A}=0.98 m c_{t-1}^{A}-0.05 m c_{t-2}^{A}+\varepsilon_{m c, t}^{A},
$$

where variables have a superscript $A$ to denote that these are artificially generated data. The

\footnotetext{
${ }^{14}$ In this exercise, OLS estimates $\widehat{\mathbf{A}}_{(s)}$ are consistent as there are no misspecification issues. The secondstage estimation can thus be characterized as asymptotic least-squares. For the properties of asymptotic least-squares, see Gouriéroux, Monfort, and Trognon (1985), and Gouriéroux and Monfort (1995, Ch. 9).

${ }^{15}$ The univariate $\operatorname{AR}(2)$ representation for real marginal costs we are using, which is given by equation (26) in the text (where a constant has been omitted), has an adjusted $R^{2}$ of 0.835 over the period 1961:Q1 to 2003:Q4. Granted, if real marginal costs are the relevant driving process for inflation, then changes to the stance of monetary policy should affect real marginal costs. In other words, one would expect the Federal funds rate to be a relevant component of the dynamics of real marginal costs. We consider in the next subsection a Monte Carlo exercise with a larger information set which also includes the Federal funds rate.

${ }^{16}$ Inflation, real marginal costs, the Federal funds rate, and GDP growth are the four variables that enter the VAR considered in Cogley and Sbordone.
} 
$\operatorname{AR}(2)$ coefficients are taken from estimating the process on actual U.S. data. With the $\operatorname{AR}(2)$ representation for real marginal costs (26), it is easy to derive the constrained reduced-form solution from which the artificial data for inflation are generated, which is given by

$$
\pi_{t}^{A}=\rho \pi_{t-1}^{A}+\frac{0.98-0.05 \beta}{1-0.98 \beta+0.05 \beta^{2}} \zeta m c_{t-1}^{A}-\frac{0.05}{1-0.98 \beta+0.05 \beta^{2}} \zeta m c_{t-2}^{A}+\zeta \varepsilon_{m c, t}^{A}+\varepsilon_{\pi, t}^{A} .
$$

This expression is a function of the vector $\psi$ of structural parameters in the NKPC.

The artificial data are generated by drawing shocks from a multivariate normal distribution, where the variance-covariance structure of the shocks is estimated on actual data given the law of motions described in (26) and (27). ${ }^{17}$ When generating the artificial data, we take zeros as initial conditions for inflation and marginal costs. This is equivalent to assuming that inflation and marginal costs are at their average levels, since we are not including constants in (26) and (27). For each Monte Carlo repetition we discard the first 500 artificially generated observations. We then estimate a reduced-form VAR of order 2 on the artificial data. ${ }^{18}$ The sample length for the VAR estimation is set at $S=176$, which corresponds to 44 years of data at quarterly frequency and is thus consistent with the sample size typically used when estimating a NKPC on actual data.

Once we have estimated the VAR coefficients matrix $\widehat{\mathbf{A}}_{(s)}$, we proceed to estimate $\widehat{\boldsymbol{\psi}}_{(s)}^{D}$ using (10), and $\widehat{\boldsymbol{\psi}}_{(s)}^{C}$ using (14). To keep matters simple, we set $\beta$ equal to 0.99 and assume that this parameter value is known and not estimated. The two parameters in $\psi$ that are left to estimate are, therefore, $\rho$ and $\zeta$. As shown earlier, $\zeta$ is a function of the parameters $(\alpha, \theta, \omega, \beta, \rho)$ and only one of the three parameters $(\alpha, \theta, \omega)$ can be estimated independently. We set $\theta$ equal to 9.8 and $\omega$ equal to $0.43 .^{19}$ We thus estimate the degree of price indexation $\rho$ and the probability $\alpha$ that a firm will not be able to reset prices optimally in a given period. In generating the data, we set $\alpha$ equal to $0.588,{ }^{20}$ and consider different values for $\rho$ - specifically, $\rho=\{0.1,0.3,0.5,0.7,0.9\}-$ to assess whether the degree of indexation affects the properties of the estimated $\widehat{\boldsymbol{\psi}}_{(s)}^{D}$ and $\widehat{\boldsymbol{\psi}}_{(s)}^{C}$.

${ }^{17}$ The estimation period is 1961:Q1 to 2003:Q4. We estimate the errors in (26) and (27), with $\rho=0.5$ and $\beta$ and $\zeta$ as described later in the text. For the purpose of estimating the errors and obtaining a variancecovariance matrix of the shocks, we include a constant in (26) and in (27). The qualitative features of the Monte Carlo exercises are not affected by reasonable changes in the variance-covariance structure of the shocks used when generating the data.

${ }^{18}$ The model-consistent reduced-form coefficients matrix has zeros for the first and second lags of inflation in the marginal costs equation (26), and a zero for the second lag of inflation in the inflation equation (27). However, when estimating the reduced-form VAR on the simulated data, we are not imposing those zero restrictions on the coefficients matrix. In other words, we are assuming that the econometrician knows that the system is fully characterized by inflation and marginal costs, but the econometrician does not know that marginal costs follow a univariate $\operatorname{AR}(2)$ process.

${ }^{19}$ This is consistent with the values reported in Cogley and Sbordone (2008).

${ }^{20}$ Again, this value for $\alpha$ is consistent with the estimates reported in Cogley and Sbordone (2008). 
Results of the Monte Carlo exercises are depicted in Figures 1 and 2, which compare the distributions of $\left(\widehat{\alpha}_{(s)}^{D}, \widehat{\alpha}_{(s)}^{C}\right)$ and $\left(\widehat{\rho}_{(s)}^{D}, \widehat{\rho}_{(s)}^{C}\right)$, respectively, for different parametrizations of $\rho$. Each Monte Carlo exercise consists of 500 repetitions. It is apparent from the pictures that estimates are better centered when the NKPC is estimated in closed form, that is, under the minimization problem (14). This is especially clear when the true $\rho$ in the inflation data generating process gets closer to unity. Then the bias in estimating $\alpha$ from the DE formulation of the NKPC becomes noticeable, with an extremely large mass of estimates at $\alpha=1$. An estimated value of $\alpha$ equal to unity implies an estimate of $\zeta$ equal to zero. In other words, the DE formulation largely fails to detect that marginal costs are the driving process for inflation when the degree of indexation becomes relatively high. Instead, the corresponding distributions for the estimated $\alpha$ under the CF specification of the NKPC do not display mass at unity.

More importantly, the figures also show that the CF estimation of the NKPC produces estimates that are much more efficient. The spread between the 95 th and the 5th percentiles in the distribution of $\widehat{\alpha}^{C}$ can be three times smaller than the same spread in the distribution of $\widehat{\alpha}^{D}$. Comparing $\widehat{\rho}^{C}$ with $\widehat{\rho}^{D}$, the gain in efficiency is somewhat less pronounced but still evident, especially so when the true $\rho$ in the inflation data generating process approaches unity. For example, when the true $\rho$ is set equal to 0.7 , the spread between the 95 th and the 5 th percentiles in the distribution of $\hat{\rho}^{C}$ is less than half the same range in the distribution of $\widehat{\rho}^{D}$.

It is worth recalling that the estimated coefficients matrix $\widehat{\mathbf{A}}_{(s)}$ from the reduced-form VAR that is used for the estimation of $(\alpha, \rho)$ is the same in the two minimization problems (10) and (14). The difference in the precision of the estimates is thus only the result of the CF specification imposing model-consistent expectations about future inflation, as discussed previously.

We have mentioned in the previous section that the efficiency of the DE estimates can be improved by imposing additional constraints on inflation expectations. In particular, we have shown that the set of cross-equation restrictions in this case is

$$
\mathbf{c}_{j}^{*}(\mathbf{A}, \boldsymbol{\psi}) \equiv \mathbf{F}^{D}(\mathbf{A}, \boldsymbol{\psi}) \cdot\left(\mathbf{I}+\ldots+(\beta \mathbf{A})^{j}\right)=\underline{0}^{\prime} .
$$

Figure 3 illustrates that a small $j$ is sufficient to approach the efficiency of the closed form estimates, which corresponds to the limiting case as $j$ goes to infinity. Setting $j=4$ already generates a substantial improvement in efficiency compared to the DE estimates (which correspond to $j=0$ ). The figure also shows that in some instances the gains in efficiency from just having $j=1$ are quite large. Note that, at quarterly frequency, $j=4$ means that we are imposing model-consistent 
constraints on the evolution of expected future inflation for only one year. We find this requirement rather conservative for a model of inflation dynamics.

\subsubsection{Robustness}

We now check that the large gains in efficiency from estimating the NKPC in closed form in our baseline Monte Carlo exercise are still present when considering alternative specifications of the reduced-form dynamics for marginal costs. We illustrate two cases that we deem especially important. In the first case, the information set is still restricted to inflation and marginal costs, but we allow feedback from lagged inflation in the evolution of marginal costs over time. This is a particularly relevant case because the NKPC, as shown in (4), implies that current inflation, once controlling for the impact of lagged inflation, is a predictor of the present discounted value of current and future marginal costs. The econometrician may not observe all of the variables useful for forecasting marginal costs, but knowing inflation is enough because inflation reveals to the econometrician the forecast of the present discounted value of current and future marginal costs. Therefore, an implication of the NKPC setup is that inflation should Granger-cause marginal costs if firms have information useful for forecasting marginal costs beyond the history of that variable. To capture such a feature of the NKPC model, we now assume that, instead of following an $\operatorname{AR}(2)$ process, the reduced-form equation for marginal costs is given by

$$
m c_{t}^{A}=0.17 \pi_{t-1}^{A}+0.14 \pi_{t-2}^{A}-0.31 \pi_{t-3}^{A}+0.90 m c_{t-1}^{A}+0.14 m c_{t-2}^{A}-0.13 m c_{t-3}^{A}+\varepsilon_{m c, t}^{A} .
$$

Equation (28) constrains the sum of the coefficients on lagged inflation to sum to zero. This is done to ensure uniqueness and stability of the solution for inflation given plausible parametrizations of $\psi$. The process in (28) is data consistent once the zero-sum restriction on the coefficients for lagged inflation is imposed. ${ }^{21}$ The Monte Carlo procedure follows the same steps as before, with the modification that the estimated reduced-form VAR to retrieve $\widehat{\mathbf{A}}_{(s)}$ in each replication is now of order 3. The parametrization of $\psi$ is the same as in our baseline exercise. The results for $\rho \in\{0.1,0.3,0.5,0.7,0.9\}$ are shown in Figures 4 and 5. The distributions of $\widehat{\rho}^{C}$ and $\widehat{\alpha}^{C}$ are tighter and better centered than the corresponding distributions for $\widehat{\rho}^{D}$ and $\widehat{\alpha}^{D}$. In all, these findings are

\footnotetext{
${ }^{21}$ The process in (28) with the zero restriction on the sum of coefficients for lagged inflation was estimated over the period 1961:Q1 to 2003:Q4. There is some evidence in the data, though not overwhelming, in favor of such a specification: In an unconstrained regression of real marginal costs on three lags of inflation and three lags of marginal costs, the test of the hypothesis that the sum of coefficients on lagged inflation is different from zero has a $p$-value of 0.126 .
} 
very similar to the baseline case in which there is no feedback from lagged inflation in the dynamics of marginal costs.

Using a larger information set which, in addition to inflation and marginal costs, also includes the Federal funds rate and GDP growth, does not change the conclusion that estimates of $\alpha$ and $\rho$ obtained from the CF specification of the NKPC are better centered and more efficient than the corresponding estimates obtained from the DE specification. This is shown in Figures 6 and 7 for $\rho \in\{0.1,0.3,0.5,0.7,0.9\}$. In this exercise, real marginal costs depend only on lagged marginal costs and lagged GDP growth:

$$
m c_{t}^{A}=-0.07 d y_{t-1}^{A}+0.18 d y_{t-2}^{A}+0.16 d y_{t-3}^{A}+0.80 m c_{t-1}^{A}+0.37 m c_{t-2}^{A}-0.21 m c_{t-3}^{A}+\varepsilon_{m c, t}^{A},
$$

where $d y$ denotes GDP growth. This process is consistent with actual data once we impose the restrictions that lagged inflation and the lagged Federal funds rate do not enter the reducedform process for real marginal costs. Again, we place these restrictions to ensure uniqueness and stability of the rational expectations solution for inflation given plausible parametrizations of $\psi$. The reduced-form data generating processes for GDP growth and the Federal funds rate include three lags of each variable in the information set, and are consistent with actual data. In the

Monte Carlo procedure, we use an estimated reduced-form VAR of order 3 to retrieve $\widehat{\mathbf{A}}_{(s)}$ in each replication. The estimation results, overall, are very similar to the results obtained in the baseline exercise.

\subsubsection{Misspecification of the Indexation Mechanism}

We consider here a particular form of misspecification in the estimation of the NKPC. The true NKPC is now given by the following expression

$$
\pi_{t}=\rho\left(\tau \pi_{t-1}+(1-\tau) \pi_{t-2}\right)+\beta\left(E_{t} \pi_{t+1}-\rho\left(\tau \pi_{t}+(1-\tau) \pi_{t-1}\right)\right)+\zeta m c_{t}+u_{t}
$$

In this NKPC setup, firms that do not reset their prices optimally in a given period follow an indexation mechanism which is not based on last period's inflation only, but on a weighted average of inflation over the past 2 periods, where $0 \leq \tau \leq 1$ denotes the weight placed on last period's inflation.

The reason for considering such a specification is that estimating the inflation process as a function of two lags of inflation and two lags of real marginal costs over the period 1961:Q1 to 
2003:Q4 yields the following estimates

$$
\pi_{t}=0.51 \pi_{t-1}+0.36 \pi_{t-2}+0.096 m c_{t-1}-0.078 m c_{t-2}+\varepsilon_{\pi, t}
$$

where standard errors are in parenthesis. ${ }^{22}$ The second lag of inflation is highly significant and, while not as large as the first lag, economically relevant. This result, together with the fact that lags of inflation are not especially important in an estimated reduced-form equation for real marginal costs over the same period, raises the possibility that a NKPC specification as in (29) provides a better characterization of the data than the specification in (1), which constrains $\tau$ to unity.

We investigate the misspecification bias that arises when the data generating process for inflation follows a NKPC as in (29) with $\tau<1$, but one estimates a NKPC, either in the DE or in the CF version, with $\tau$ constrained to equal unity. For this purpose, we set up a Monte Carlo exercise which is very similar to our baseline exercise in section 2.3.1. Inflation and marginal costs are the only two variables in the information set. Real marginal costs follow the same reduced-form $\operatorname{AR}(2)$ process as in (26). In generating the data, we set $\tau$ equal to 0.6. The other parameters $(\alpha, \beta, \theta, \omega)$ are set as before, and we consider different values for $\rho$. The VAR used to retrieve the matrix $\widehat{\mathbf{A}}_{(s)}$ in each Monte Carlo replication is of order 2. The misspecification in this exercise arises from the fact that the estimated NKPC, both in the DE and the CF specifications, constrains $\tau$ to unity.

Estimation results for $\alpha$ and $\rho$, with $\rho=\{0.1,0.3,0.5,0.7,0.9\}$, are reported in Figures 8 and 9, which compare the distributions of $\left(\widehat{\alpha}_{(s)}^{D}, \widehat{\alpha}_{(s)}^{C}\right)$ and $\left(\widehat{\rho}_{(s)}^{D}, \widehat{\rho}_{(s)}^{C}\right)$ for the different parametrizations of $\rho$. It is apparent that when the the NKPC is estimated in the DE form via the minimum-distance problem (10), the estimates $\left(\widehat{\alpha}^{D}, \widehat{\rho}^{D}\right)$ are biased. The estimate for $\alpha$ is upward biased, with the estimation procedure often failing to identify real marginal costs as the driving process for inflation. The estimate for $\rho$ is downward biased. In other words, the estimates point to less indexation to past inflation than is actually present in the true data generating process. When the NKPC is estimated in closed form via the minimum-distance problem (14), estimates for $\alpha$ are well centered. There is some downward bias, instead, when estimating $\rho$. However, the bias is not as large as with the DE specification, as the figure clearly shows.

\footnotetext{
${ }^{22}$ Augmenting the information set to include lagged GDP growth and lagged policy rates does not materially alter the estimates of the coefficients on lagged inflation in (30). The median estimates of the two lags of inflation in the inflation equation from the reduced-form VAR used by Cogley and Sbordone, which is estimated with Bayesian methods, are 0.40 and 0.32 respectively.
} 
In all, the results in this section highlight the importance of correctly specifying the indexation rule in the NKPC. This is true both in the DE and in the CF specifications of the NKPC, though it is apparent that the $\mathrm{CF}$ version is less prone to suffer from this misspecification bias than the DE counterpart.

\section{Estimates of the NKPC with Time-Varying Trend Inflation}

Given our Monte Carlo findings, we now turn to estimating a NKPC with time-varying trend inflation on actual data. We first provide a brief description of the DE and the CF representations of the NKPC in this setup, and then describe the estimation method and the empirical findings.

\subsection{Model Setup}

The framework is the same used by Cogley and Sbordone, with the exception that we allow the indexation mechanism to depend on two lags of inflation. Specifically, we have

$$
P_{t}(i)=\left(\Pi_{t-1}^{\tau} \Pi_{t-2}^{1-\tau}\right)^{\rho} P_{t-1}(i)
$$

where $P_{t}(i)$ is the price set by firm $i$ when it cannot reoptimize at time $t$, and $\Pi_{t}=P_{t} / P_{t-1}$ is the period $t$ gross rate of inflation. In this expression, and as in section $2, \rho \in[0,1]$ measures the degree of indexation, while $\tau \in[0,1]$ represents the weight given to $t-1$ aggregate inflation relative to $t-2$ aggregate inflation. This indexation mechanism is more general than Cogley and Sbordone's, and it nests their setup as a special case when $\tau=1$. Cogley and Sbordone characterize the dynamics of inflation when trend (steady-state) inflation is time-varying. As such, the setup differs from the simple NKPC equation of the previous sections, which was derived by log-linearizing the first-order conditions of the Calvo pricing model around a zero-inflation steady-state. The log-linearization is now taken around a steady-state where trend inflation changes over time. It is assumed that trend inflation is an exogenous process that evolves as a random walk. The distinguishing feature of this type of setup, compared to the more standard setup with zero trend inflation, is that the coefficients in the NKPC are a function of trend inflation and as a result they are time-varying.

Since our departure from Cogley and Sbordone's setup is very minor, we leave the full details of the derivation of the NKPC to appendices A and B. In the rest of this section, we provide the equilibrium relationships of the model with time-varying trend inflation that we use at the 
estimation stage. The first of these relationships is the restriction between trend inflation and steady-state real marginal costs, which as in Cogley and Sbordone takes the form

$$
\left(1-\alpha \bar{\Pi}_{t}^{(1-\rho)(\theta-1)}\right)^{(1+\theta \omega) /(1-\theta)}\left[\frac{1-\alpha \overline{q g} y \bar{\Pi}_{t}^{\theta(1+\omega)(1-\rho)}}{1-\alpha \overline{q g} y \bar{\Pi}_{t}^{(\theta-1)(1-\rho)}}\right]=(1-\alpha)^{(1+\theta \omega) /(1-\theta)} \frac{\theta}{\theta-1} \overline{m c}_{t},
$$

where $\bar{q}$ is the steady-state real discount factor, $\bar{g}^{y}$ is the steady-state growth rate of output, $\bar{\Pi}$ is gross trend inflation, $\overline{m c}$ steady-state real marginal costs, and the other parameters are defined in the previous sections. Denoting by a hat the log-deviation of a variable from its steady-state value, we can write the NKPC as follows

$\widehat{\pi}_{t}=\rho \tau\left(\widehat{\pi}_{t-1}-\widehat{g}_{t}^{\pi}\right)+\rho(1-\tau)\left(\widehat{\pi}_{t-1}-\widehat{g}_{t-1}^{\pi}-\widehat{g}_{t}^{\pi}\right)+\lambda_{t} E_{t}\left(\widehat{\pi}_{t+1}-\rho \tau \widehat{\pi}_{t}-\rho(1-\tau)\left(\widehat{\pi}_{t-1}-\widehat{g}_{t}^{\pi}\right)\right)+\zeta_{t} \widehat{m c}_{t}+\gamma_{t} \widehat{D}_{t}$

where $\widehat{g}_{t}^{\pi}=\ln \left(\bar{\Pi}_{t} / \bar{\Pi}_{t-1}\right)$ is the growth rate of trend inflation and $\widehat{D}$ is defined recursively as

$$
\widehat{D}_{t}=\varphi_{1 t} E_{t}\left(\widehat{q}_{t, t+1}+\widehat{g}_{t+1}^{y}\right)+\varphi_{1 t}(\theta-1) E_{t}\left\{\widehat{\pi}_{t+1}-\rho \tau \widehat{\pi}_{t}-\rho(1-\tau)\left(\widehat{\pi}_{t-1}-\widehat{g}_{t}^{\pi}\right)\right\}+\varphi_{1 t} E_{t} \widehat{D}_{t+1} .
$$

Some coefficients in (32) and (33) are time-varying. Compared to the standard NKPC specification with zero trend inflation, this specification entails, in addition to the growth rate in trend inflation, other terms summarized by $\widehat{D}$. It is possible to iterate forward equation (32) to obtain an infinitehorizon specification that takes the form

$$
\widehat{\pi}_{t}=\rho \tau\left(\widehat{\pi}_{t-1}-\widehat{g}_{t}^{\pi}\right)+\rho(1-\tau)\left(\widehat{\pi}_{t-1}-\widehat{g}_{t-1}^{\pi}-\widehat{g}_{t}^{\pi}\right)+\zeta_{t} \sum_{i=0}^{\infty} \lambda_{t}^{i} E_{t} \widehat{m c}_{t+i}+\gamma_{t} \sum_{i=0}^{\infty} \lambda_{t}^{i} E_{t} \widehat{D}_{t+i}
$$

which we refer to as the closed-form $(\mathrm{CF})$ version of the NKPC. ${ }^{23}$ The expression in (34) is not entirely "closed-form" in that $\widehat{D}_{t}$ is a function of past, current, and expected future inflation, as (33) shows. Forward iteration of (33) also indicates that obtaining a closed-form representation for inflation in this setup is complicated unless indexation is full $(\rho=1)$ or steady-state inflation is zero. Still, the quasi closed form in (34) imposes model-consistent expectations on the process followed by future inflation that are not imposed in the DE form (32). These restrictions on expected inflation are of the same kind as the restrictions imposed by the CF specification (4) relative to DE form (2) in the standard constant coefficients setup with zero trend-inflation.

\footnotetext{
${ }^{23}$ In deriving (34), we use the "anticipated utility" assumption that $E_{t} \zeta_{t+i} \prod_{k=0}^{i} \lambda_{t+k} \widehat{m c} c_{t+i}=\zeta_{t} \lambda_{t}^{i+1} E_{t} \widehat{m c}_{t+i}$ and $E_{t} \gamma_{t+i} \prod_{k=0}^{i} \lambda_{t+k} \widehat{D}_{t+i}=\gamma_{t} \lambda_{t}^{i+1} E_{t} \widehat{D}_{t+i}$ for any $i>0$, as in Cogley and Sbordone.
} 


\subsection{Estimation Approach}

We want to compare estimates of the deep structural parameters $\psi=(\alpha, \rho, \theta, \tau)$ obtained from the DE form (32) with the estimates obtained from the CF representation (34). The econometric approach has already been discussed in section 2.1 and replicates Cogley and Sbordone. Forecasts of the relevant variables pertaining to the NKPC are obtained by means of a reduced-form VAR. In the present context in which trend inflation is time-varying, the reduced-form VAR has drifting coefficients. The first-order form of the VAR can then be written as

$$
\mathbf{z}_{t}=\boldsymbol{\mu}_{t}+\mathbf{A}_{t} \mathbf{z}_{t-1}+\varepsilon_{z, t}
$$

where the coefficients in $\boldsymbol{\mu}_{t}$ and $\mathbf{A}_{t}$ are assumed to evolve as a random walk. The evolution of the coefficients in $\mathbf{A}_{t}$ is constrained by reflecting barriers, so that the roots of $\mathbf{A}_{t}$ at each point in time lie inside the unit circle. The conditional expectation of a variable $y_{t+k}$ at time $t-2$ is then given by

$$
E_{t-2} y_{t+k}=\mathbf{e}_{y}^{\prime} \mathbf{A}_{t-2}^{k+2}\left(\mathbf{z}_{t-2}-\boldsymbol{\mu}_{t-2}\right),
$$

where $\mathbf{e}_{y}^{\prime}$ is the selection vector for variable $y_{t-2}$ in $\mathbf{z}_{t-2}$.

Given the forecasting rule (36) and equations (32) and (33), we obtain the conditional expectation of inflation based on information at $t-2$ in the DE form as follows

$$
\begin{aligned}
\mathbf{e}_{\pi}^{\prime} \mathbf{A}_{t-2}^{2} \widehat{\mathbf{z}}_{t-2}= & \widetilde{\rho}_{1, t-2}^{D} \mathbf{e}_{\pi}^{\prime} \mathbf{A}_{t-2} \widehat{\mathbf{z}}_{t-2}+(1-\tau) \widetilde{\rho}_{2, t-2}^{D} \mathbf{e}_{\pi}^{\prime} \widehat{\mathbf{z}}_{t-2}+\widetilde{\zeta}_{t-2}^{D} \mathbf{e}_{m c}^{\prime} \mathbf{A}_{t-2}^{2} \widehat{\mathbf{z}}_{t-2} \\
& +d_{1 t-2}^{D} \mathbf{e}_{\pi}^{\prime} \mathbf{A}_{t-2}^{3} \widehat{\mathbf{z}}_{t-2}+d_{2 t-2}^{D} \varphi_{1 t-2} \mathbf{e}_{\pi}^{\prime} \mathbf{J}_{t-2} \mathbf{A}_{t-2}^{4} \widehat{\mathbf{z}}_{t-2} \\
& +d_{3 t-2}^{D}\left(\mathbf{e}_{Q}^{\prime} \mathbf{J}_{t-2} \mathbf{A}_{t-2}^{2} \widehat{\mathbf{z}}_{t-2}+\mathbf{e}_{g^{y}}^{\prime} \mathbf{J}_{t-2} \mathbf{A}_{t-2}^{3} \widehat{\mathbf{z}}_{t-2}\right),
\end{aligned}
$$

where

$$
\mathbf{J}_{t} \equiv\left(\mathbf{I}-\varphi_{1 t} \mathbf{A}_{t}\right)^{-1}
$$

The full derivation of (37) and the definition of coefficients is left to the appendix. We take expectations as of $t-2$ because the indexation mechanism is based on two lags of inflation, and this allows to ignore the terms involving the growth rate of trend inflation. ${ }^{24}$ As assumed by Cogley and Sbordone, expectations are formed using an "anticipated utility" framework (Kreps, 1998, and Cogley and Sargent, 2008), where at each point in time agents expect all model coefficients to stay

\footnotetext{
${ }^{24} \mathrm{As}$ already mentioned, trend inflation evolves as a (driftless) random walk, and therefore the expected future growth rate of trend inflation is zero.
} 
constant at their current values going forward. The vector of cross-equation restrictions implied by the conditional expectation in (37) is then

$$
\begin{aligned}
\mathbf{e}_{\pi}^{\prime} \mathbf{A}_{t-2}^{2}= & \widetilde{\rho}_{1, t-2}^{D} \mathbf{e}_{\pi}^{\prime} \mathbf{A}_{t-2}+(1-\tau) \widetilde{\rho}_{2, t-2}^{D} \mathbf{e}_{\pi}^{\prime} \mathbf{I}+\widetilde{\zeta}_{t-2}^{D} \mathbf{e}_{m c}^{\prime} \mathbf{A}_{t-2}^{2} \\
& +d_{1 t-2}^{D} \mathbf{e}_{\pi}^{\prime} \mathbf{A}_{t-2}^{3}+d_{2 t-2}^{D} \varphi_{1 t-2} \mathbf{e}_{\pi}^{\prime} \mathbf{J}_{t-2} \mathbf{A}_{t-2}^{4} \\
& +d_{3 t-2}^{D}\left(\mathbf{e}_{Q}^{\prime} \mathbf{J}_{t-2} \mathbf{A}_{t-2}^{2}+\mathbf{e}_{g^{y}}^{\prime} \mathbf{J}_{t-2} \mathbf{A}_{t-2}^{3}\right) \\
\equiv & \mathbf{g}^{D}\left(\boldsymbol{\mu}_{t-2}, \mathbf{A}_{t-2}, \boldsymbol{\psi}\right)
\end{aligned}
$$

and the distance between expected inflation as of $t-2$ and model-consistent inflation is given by

$$
\mathbf{F}_{1}^{D}\left(\boldsymbol{\mu}_{t-2}, \mathbf{A}_{t-2}, \boldsymbol{\psi}\right) \equiv \mathbf{e}_{\pi}^{\prime} \mathbf{A}_{t-2}^{2}-\mathbf{g}^{D}\left(\boldsymbol{\mu}_{t-2}, \mathbf{A}_{t-2}, \boldsymbol{\psi}\right)=\underline{0^{\prime}}
$$

In contrast, for the CF specification of the NKPC in (34) the conditional expectation of inflation based on information at $t-2$ is now

$$
\begin{aligned}
\mathbf{e}_{\pi}^{\prime} \mathbf{A}_{t-2}^{2} \widehat{\mathbf{z}}_{t-2}= & \widetilde{\rho}_{1, t-2}^{C} \mathbf{e}_{\pi}^{\prime} \mathbf{A}_{t-2} \widehat{\mathbf{z}}_{t-2}+(1-\tau) \widetilde{\rho}_{2, t-2}^{C} \mathbf{e}_{\pi}^{\prime} \widehat{\mathbf{z}}_{t-2}+\widetilde{\zeta}_{t-2}^{C} \mathbf{e}_{m c}^{\prime} \mathbf{K}_{t-2} \mathbf{A}_{t-2}^{2} \widehat{\mathbf{z}}_{t-2} \\
& +d_{0 t-2}^{C} \mathbf{e}_{\pi}^{\prime} \mathbf{K}_{t-2} \mathbf{A}_{t-2} \widehat{\mathbf{z}}_{t-2}+d_{1 t-2}^{C} \mathbf{e}_{\pi}^{\prime} \mathbf{K}_{t-2} \mathbf{A}_{t-2}^{2} \widehat{\mathbf{z}}_{t-2} \\
& +d_{2 t-2}^{C} \mathbf{e}_{\pi}^{\prime} \mathbf{K}_{t-2} \mathbf{A}_{t-2}^{3} \widehat{\mathbf{z}}_{t-2}+d_{2 t-2}^{C} \varphi_{1 t-2} \mathbf{e}_{\pi}^{\prime} \mathbf{K}_{t-2} \mathbf{J}_{t-2} \mathbf{A}_{t-2}^{4} \widehat{\mathbf{z}}_{t-2} \\
& +d_{3 t-2}^{C}\left(\mathbf{e}_{Q}^{\prime} \mathbf{K}_{t-2} \mathbf{J}_{t-2} \mathbf{A}_{t-2}^{2} \widehat{\mathbf{z}}_{t-2}+\mathbf{e}_{g^{y}}^{\prime} \mathbf{K}_{t-2} \mathbf{J}_{t-2} \mathbf{A}_{t-2}^{3} \widehat{\mathbf{z}}_{t-2}\right)
\end{aligned}
$$

where

$$
\mathbf{K}_{t} \equiv\left(\mathbf{I}-\lambda_{t} \mathbf{A}_{t}\right)^{-1}
$$

We leave again the derivation of (39) and the definition of coefficients to the appendix. The vector of cross-equation restrictions implied by the conditional expectation in (34) is then given by

$$
\begin{aligned}
\mathbf{e}_{\pi}^{\prime} \mathbf{A}_{t-2}^{2}= & \widetilde{\rho}_{1, t-2}^{C} \mathbf{e}_{\pi}^{\prime} \mathbf{A}_{t-2}+(1-\tau) \widetilde{\rho}_{2, t-2}^{C} \mathbf{e}_{\pi}^{\prime} \mathbf{I}+\widetilde{\zeta}_{t-2}^{C} \mathbf{e}_{m c}^{\prime} \mathbf{K}_{t-2} \mathbf{A}_{t-2}^{2} \\
& +d_{0 t-2}^{C} \mathbf{e}_{\pi}^{\prime} \mathbf{K}_{t-2} \mathbf{A}_{t-2}+d_{1 t-2}^{C} \mathbf{e}_{\pi}^{\prime} \mathbf{K}_{t-2} \mathbf{A}_{t-2}^{2} \\
& +d_{2 t-2}^{C} \mathbf{e}_{\pi}^{\prime} \mathbf{K}_{t-2} \mathbf{A}_{t-2}^{3}+d_{2 t-2}^{C} \varphi_{1 t-2} \mathbf{e}_{\pi}^{\prime} \mathbf{K}_{t-2} \mathbf{J}_{t-2} \mathbf{A}_{t-2}^{4} \\
& +d_{3 t-2}^{C}\left(\mathbf{e}_{Q}^{\prime} \mathbf{K}_{t-2} \mathbf{J}_{t-2} \mathbf{A}_{t-2}^{2}+\mathbf{e}_{g^{y}}^{\prime} \mathbf{K}_{t-2} \mathbf{J}_{t-2} \mathbf{A}_{t-2}^{3}\right) \\
\equiv & \mathbf{g}^{C}\left(\boldsymbol{\mu}_{t-2}, \mathbf{A}_{t-2}, \boldsymbol{\psi}\right) .
\end{aligned}
$$

As in the one-period ahead formulation, the relevant distance for the estimation is

$$
\mathbf{F}_{1}^{C}\left(\boldsymbol{\mu}_{t}, \mathbf{A}_{t}, \boldsymbol{\psi}\right) \equiv \mathbf{e}_{\pi}^{\prime} \mathbf{A}_{t}^{2}-\mathbf{g}^{C}\left(\boldsymbol{\mu}_{t}, \mathbf{A}_{t}, \boldsymbol{\psi}\right)=\underline{0}^{\prime}
$$


The function $\mathbf{g}^{C}\left(\boldsymbol{\mu}_{t}, \mathbf{A}_{t}, \psi\right)$ imposes model-consistent constraints that are not captured in $\mathbf{g}^{D}\left(\boldsymbol{\mu}_{t}, \mathbf{A}_{t}, \psi\right)$. The intuition behind these additional constraints is the same as in the constant coefficients formulation of the NKPC examined in section 2.

When trend inflation varies over time, the long-run relationship given by equation (31) provides an additional constraint at the estimation stage, for both the $\mathrm{DE}$ and $\mathrm{CF}$ specifications. Correspondingly, we define

$\mathbf{F}_{2}\left(\boldsymbol{\mu}_{t}, \mathbf{A}_{t}, \boldsymbol{\psi}\right)=\left(1-\alpha \bar{\Pi}_{t}^{(1-\rho)(\theta-1)}\right)^{(1+\theta \omega) /(1-\theta)}\left[\frac{1-\alpha \overline{q g} y \bar{\Pi}_{t}^{\theta(1+\omega)(1-\rho)}}{1-\alpha \overline{q g}^{y} \bar{\Pi}_{t}^{(\theta-1)(1-\rho)}}\right]-(1-\alpha)^{(1+\theta \omega) /(1-\theta)} \frac{\theta}{\theta-1} \overline{m c}_{t}=\underline{0}^{\prime}$.

The complete set of cross equation restrictions to be satisfied at each point in time is then given by

$$
\begin{array}{rlr}
\mathcal{F}_{t}^{j} & \equiv\left[\mathbf{F}_{1}^{j}\left(\boldsymbol{\mu}_{t}, \mathbf{A}_{t}, \boldsymbol{\psi}\right), \mathbf{F}_{2}\left(\boldsymbol{\mu}_{t}, \mathbf{A}_{t}, \boldsymbol{\psi}\right)\right] & \\
\mathcal{F}^{j}(\boldsymbol{\Theta}) & \equiv\left[\mathcal{F}_{1}^{j \prime}, \ldots, \mathcal{F}_{T}^{j \prime}\right]^{\prime} & \text { for } j \epsilon\{D, C\},
\end{array}
$$

where

$$
\Theta=\left\{\boldsymbol{\mu}_{t}, \mathbf{A}_{t}\right\}_{t=1}^{T}
$$

As in the constant coefficients case, estimation is a two step procedure. Now we first use Bayesian methods to characterize the posterior distribution of $\boldsymbol{\Theta}$ from a set of $M$ estimates $\widehat{\boldsymbol{\Theta}}=\left\{\widehat{\boldsymbol{\Theta}}_{i}\right\}_{i=1}^{M}$. In the second step we obtain the structural parameter estimates

$$
\widehat{\boldsymbol{\psi}}_{i}^{D}=\arg \min \mathcal{F}^{D}\left(\widehat{\boldsymbol{\Theta}}_{i}\right)^{\prime} \cdot \mathcal{F}^{D}\left(\widehat{\boldsymbol{\Theta}}_{i}\right) \quad \text { for } i=1, . ., M
$$

for the DE form of the NKPC, and

$$
\widehat{\boldsymbol{\psi}}_{i}^{C}=\arg \min \mathcal{F}^{C}\left(\widehat{\boldsymbol{\Theta}}_{i}\right)^{\prime} \cdot \mathcal{F}^{C}\left(\widehat{\mathbf{\Theta}}_{i}\right) \quad \text { for } i=1, \ldots, M
$$

for the CF specification.

We use the same data and Bayesian estimation procedure as Cogley and Sbordone to obtain $\widehat{\Theta} .^{25}$ The technical aspects of the estimation and the data are described in their paper. Here, we just mention that the reduced-form time-varying VAR is of order 2 and has four variables: inflation, real marginal costs (as proxied by the labor share), output growth, and a nominal discount factor based on the federal funds rate. Because the first stage Bayesian estimation yields an entire posterior distribution $\left\{\widehat{\boldsymbol{\Theta}}_{i}\right\}_{i=1}^{M}$, the second stage also provides a distribution of estimates $\left\{\widehat{\boldsymbol{\psi}}_{i}^{D}\right\}_{i=1}^{M}$ and $\left\{\widehat{\boldsymbol{\psi}}_{i}^{C}\right\}_{i=1}^{M}$.

\footnotetext{
${ }^{25}$ The code and data used by Cogley and Sbordone were retrieved from the American Economic Review website.
} 


\subsection{Estimation Results}

Table 1 displays the estimation results. ${ }^{26}$ Note that, as in Cogley and Sbordone (2008), the parameters $\beta$ and $\omega$ are not estimated and their values are set to 0.99 and 0.43 , respectively. The first specification that we consider (denoted as $D E_{-}$const in the table) replicates the baseline estimates of Cogley and Sbordone and yields their same median estimates. This corresponds to the DE es-

timates $\widehat{\boldsymbol{\psi}}^{D}$ with $\tau$ constrained to equal 1. The indexation parameter $\rho$ is estimated at zero, and the estimated $\alpha$ implies that the median span of time between optimal price resets is 3.9 months.

We then consider the DE specification without constraining $\tau$ to equal unity. This allows the indexation mechanism to depend not just on the first lag, but also on the second lag of inflation. As already mentioned, this is an important check on the specification as the reduced-form inflation equation from the estimated VAR places a significant weight on the second lag of inflation. Our Monte Carlo results in section 2.3 illustrate that the misspecification bias from incorrectly constraining $\tau$ to unity can be very large, especially so when the NKPC is estimated in the DE form as in Cogley and Sbordone. Estimation results (denoted as DE_ unconst in the table) show that this simple modification produces a very different estimate for $\rho$, suggesting misspecification bias when estimating the NKPC with $\tau$ set to 1 . The median estimated value for $\rho$ jumps from zero in the previous case to 0.63 . While imprecisely estimated, the 90 percent confidence interval does not include zero. Moreover, $\tau$ is estimated at 0.56 , and its 90 percent confidence interval is bounded well away from one. This implies that the first and the second lags of inflation in the indexation mechanism receive approximately the same weight.

Next we consider the estimates obtained from the CF specification of the NKPC. To highlight the differences in results when this specification is used instead of the DE form, we report constrained $(\tau=1)$ and unconstrained estimates. For the constrained case $\left(C F_{-}\right.$const in the table) the median estimate of $\rho$ is 0.51 , with 90 percent confidence bands that do not encompass zero. Note also that the median estimate of $\alpha$ increases to 0.80 , implying a median time between price re-optimization of 9.3 months, more than twice the estimate obtained from the DE specification. Moreover, the 90 percent confidence intervals for this parameter in the DE and CF specifications have negligible overlap.

Estimating the CF specification that does not constrain $\tau$ ( $C F_{-}$unconst in the table) yields a median estimate of $\rho$ equal to 0.88 . This is an even larger estimate than the one obtained from

\footnotetext{
${ }^{26}$ We report as point estimate the median of the distribution for each parameter.
} 
the DE counterpart, and it is estimated much more precisely. The median estimate of $\alpha$, at 0.86 , implies that re-optimization now occurs every 14.1 months. The indexation mechanism places a larger weight on the first lag of inflation than in the DE specification; the median estimate is now 0.68. Still, the 90 percent confidence interval for $\tau$ does not contain unity. The median estimate of $\theta$ is 12.6 , which implies a steady-state markup of 8.6 percent. This estimate for the steadystate markup is somewhat lower than the steady-state markup of 11 percent estimated from the corresponding DE specification. In addition, the 90 percent confidence interval for $\theta$ tends to be larger using the CF specification, suggesting that when all of the model restrictions on expectations are taken into account the link from marginal costs to inflation becomes somewhat more uncertain.

In figure 10 we illustrate the arguments discussed in section 2.2 regarding the effects of adding model-consistent constraints on expected inflation when estimating the DE form (with $\tau$ unconstrained). In the figure, the horizontal axis indicates the number of additional periods $j$ during which expected future inflation is constrained to evolve in a model-consistent way. Similar to our Monte Carlo findings, the figure shows that setting $j=4$ results in a median estimate that is already remarkably close to the CF estimate and much more precise than the original DE estimate $(j=0$ in the figure).

\subsection{Robustness Checks and Discussion of Results}

We check that the estimated distributions for $\alpha, \rho$, and $\tau$ for the CF case do not change substantially when we discard the estimates for $\alpha, \rho$, and $\tau$ associated with estimated values of $\theta$ above $40 .{ }^{27}$ The results of this exercise are reported in Table 2. The 90 percent confidence interval for the remaining estimates of $\theta$ is substantially reduced, while the median estimate (at 12.4) remains virtually the same. Changes in median estimates and confidence bands for the other parameters are negligible. We also considered versions of the NKPC which omit terms involving the discount factor, output growth and terms involving higher order leads of inflation. Estimation results for the same four specifications discussed in the previous section are reported in Table C1 in appendix C. In comparing the estimates from the $\mathrm{DE}$ and $\mathrm{CF}$ specifications, the main conclusions from the previous section remain unaltered. The only results worth mentioning are that $(i)$ the 90 percent confidence bands for $\theta$ are now much tighter when estimating the CF specification, and ( $i i$ ) the estimated $\theta$ is now very similar across different specifications.

\footnotetext{
${ }^{27}$ This represents roughly the top decile of the distribution of $\theta$ in the CF estimates.
} 
In all, the estimation findings from the CF specification of the NKPC with two lags of inflation in the indexation mechanism, which we favor on the basis of the Monte Carlo simulations from section 2.3, are noticeably different from the estimates obtained from the DE specification with $\tau$ set to 1 . The results indicate that lagged inflation plays an important role in explaining inflation dynamics even when controlling for time-varying trend inflation. When interpreting these findings, note that having $\rho$ equal to 1 implies that the NKPC (expressed in the DE form) assigns about the same weights to lagged inflation and to expected future inflation. ${ }^{28}$

Our estimate of $\rho$ from the closed-form representation of the NKPC is similar to estimates obtained previously in the literature, albeit using different methods and mostly without considering trend inflation. An even split between past and future inflation when characterizing inflation dynamics in the NKPC appears to be common to those estimation procedures which, like ours, take explicitly into account the constraints placed by the NKPC on all future expectations of inflation (Fuhrer and Moore, 1995, Fuhrer and Olivei, 2005). ${ }^{29}$ Still, it is important to stress that in our setup expectations are formed using the same reduced-form VAR in both the DE and the CF specifications of the NKPC. In this sense, the CF specification does not use more information at the estimation stage compared to the DE form, nor it uses different equations to complete the model. The only additional information embedded in the closed form of the NKPC are the model-consistent constraints placed on future expectations.

The autocorrelation properties of detrended inflation are one important dimension of the data that are better captured by a high value of $\rho$. After removing the time-varying trend, the first autocorrelation of inflation in the data over the period 1960:Q3 to 2003:Q4 is equal to 0.81. Fuhrer (2009) notes that with $\rho$ equal to zero the first autocorrelation implied by the NKPC is very low, and argues that a $\rho$ close to one is necessary to match this crucial feature of the data.

\section{Conclusions}

We show that estimates of the deep parameters in the New Keynesian Phillips curve (NKPC) can differ substantially when the NKPC is estimated in the difference equation (DE) form versus the closed form ( $\mathrm{CF})$. In Monte Carlo simulations, we illustrate how the addition of model-consistent

\footnotetext{
${ }^{28}$ This case would correspond to the NKPC considered in Christiano, Eichenbaum, and Evans (2005).

${ }^{29}$ The finding of a $\rho$ significantly different from zero is also consistent with work by Kozicki and Tinsley (2002) which explicitly considers time-varying trend inflation.
} 
constraints embedded in the closed form (which do not necessarily hold in the DE form) yields to more efficient and less biased estimates. On actual data, moving from the DE specification (Cogley and Sbordone 2008) to the CF version of the NKPC yields different conclusions about the importance of lagged inflation in the NKPC. The estimate for the indexation parameter $\rho$ goes from zero in the DE form to almost one using the CF specification, thus assigning almost the same weight to lagged inflation and expected future inflation. The estimation of the closed form thus suggests that accounting for time-varying trend inflation does not eliminate the need for lags of inflation in the NKPC. To some extent, this result holds for the estimates based on the DE specification when we consider a richer indexation mechanism. In addition, the CF specification implies that prices are re-optimized much less frequently than what is suggested by the DE form (approximately 12 months versus 4 months).

The Monte Carlo findings give reasons to prefer the estimates from the CF specification. However, these results come from an exercise in which the data are generated from the solution to the NKPC model. In essence, the constraints imposed by the CF specification of the NKPC hold by construction. It is not clear which form one would prefer to use once the data-generating process is not exactly the NKPC model. For example, it is possible that if agents do not form expectations about future inflation in a manner that is consistent with the NKPC holding at each future point in time, relying on the less-constrained DE form could prevent a source of misspecification bias. However, it is not necessary for expectations to be model-consistent ad infinitum. A modest amount of discipline on expectations (roughly one year) already closes most of the gap between $\mathrm{DE}$ and CF estimates. Moreover, there is no reason to argue that misspecification biases would always penalize the CF specification more than the DE form. We have discussed one form of misspecification concerning the indexation lags that strongly biases the results in the DE form but has much less impact on the CF estimates, both in a Monte Carlo exercise and in actual data. Finally, even abstracting from misspecification issues, the CF estimates are better able to capture the autocorrelation properties of the deviations of inflation from the time-varying trend inflation. These deviations are highly autocorrelated in the data, and this fact is hard to reconcile with a NKPC that is purely forward-looking. Future research should explore the sensitivity of the estimates of the deep parameters in the NKPC to variations in the estimation period, given some evidence that inflation persistence has declined in the most recent years. 
Figure 1: Monte Carlo simulations, estimates of $\alpha$ Difference equation (DE) vs. closed-form (CF)

$\mathrm{DE}$
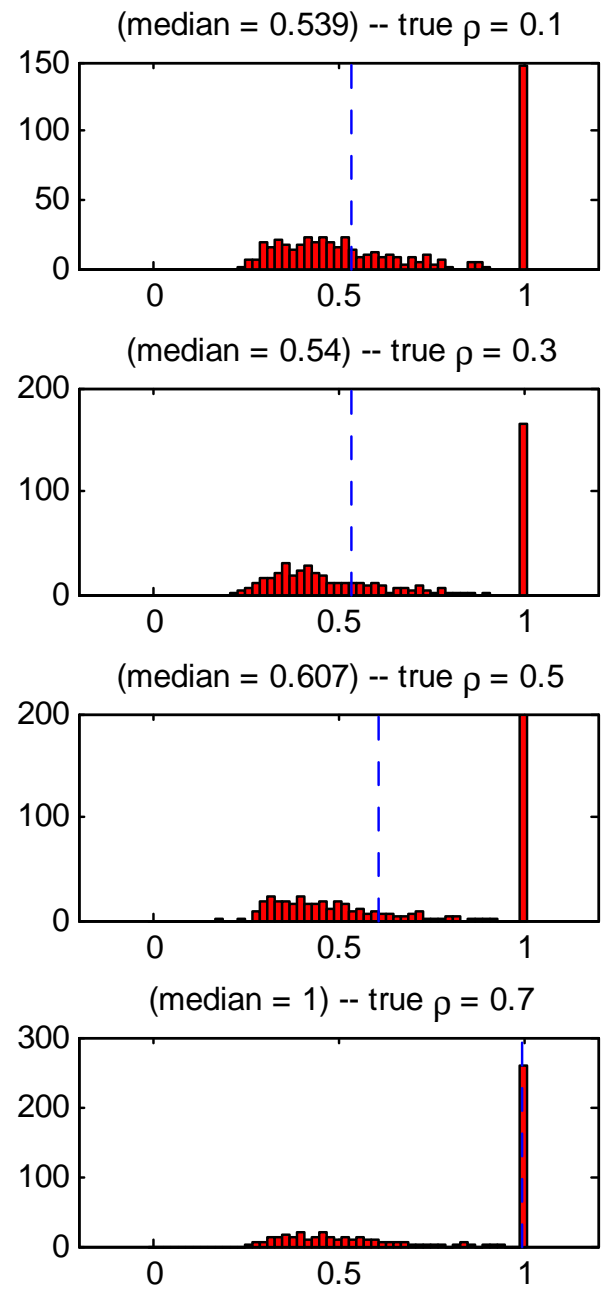

$($ median $=1)$-- true $\rho=0.9$

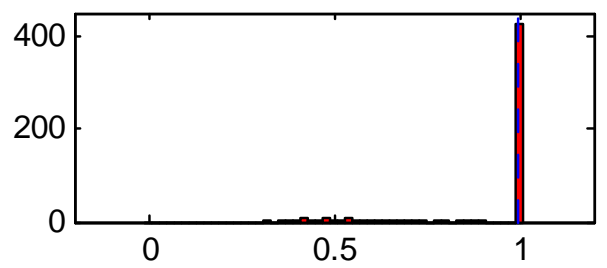

CF
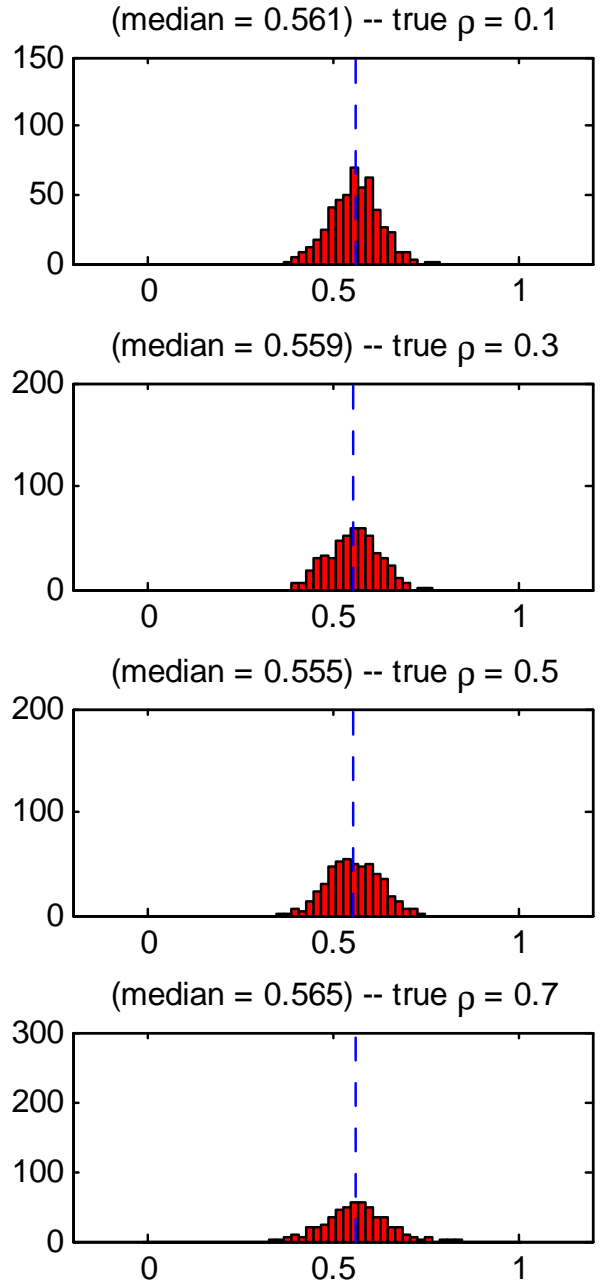

(median $=0.544)$-- true $\rho=0.9$

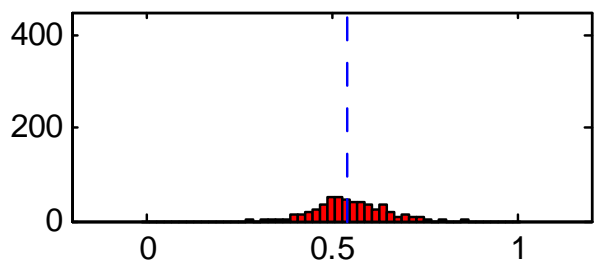

Notes:

(1) left and right panels correspond to DE and CF specifications, respectively;

(2) the vertical axis is the number of repetitions. Total number of repetitions is 500 . 
Figure 2: Monte Carlo simulations, estimates of $\rho$ Difference equation (DE) vs. closed-form (CF)

DE
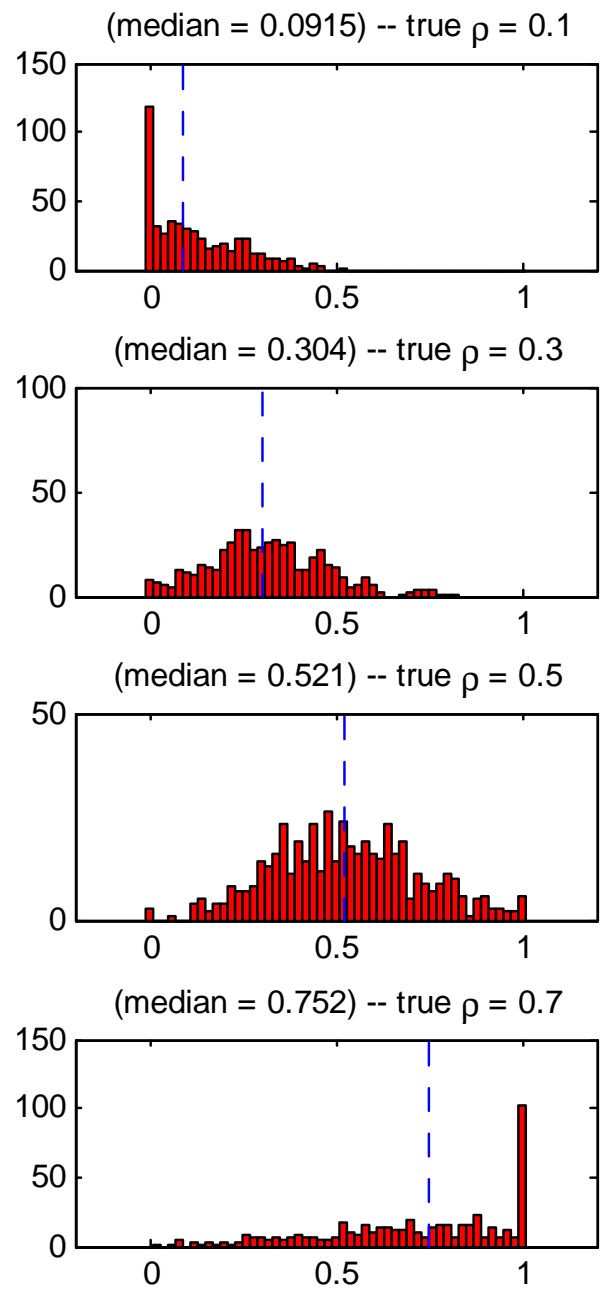

$($ median $=0.904)--$ true $\rho=0.9$

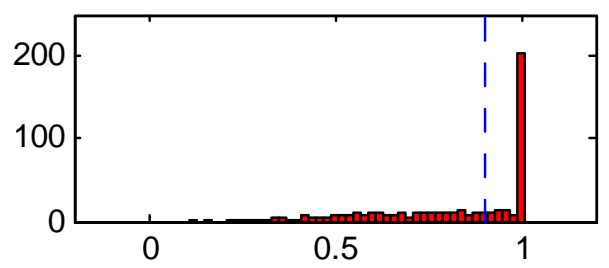

CF
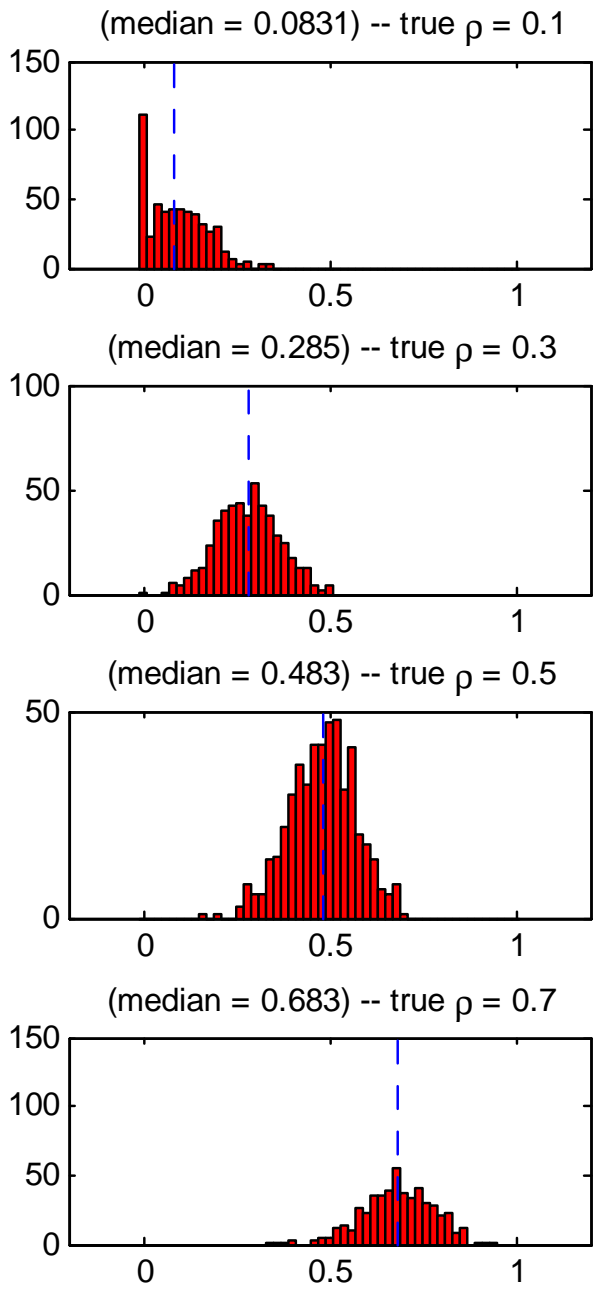

(median $=0.9)$-- true $\rho=0.9$

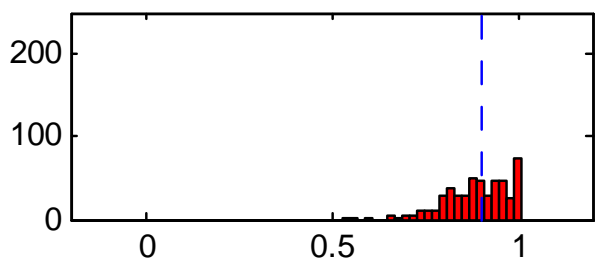

Notes:

(1) left and right panels correspond to DE and CF specifications, respectively;

(2) the vertical axis is the number of repetitions. Total number of repetitions is 500 . 
Figure 3: 90\% confidence interval width after imposing additional expectational restrictions* (Monte Carlo results)
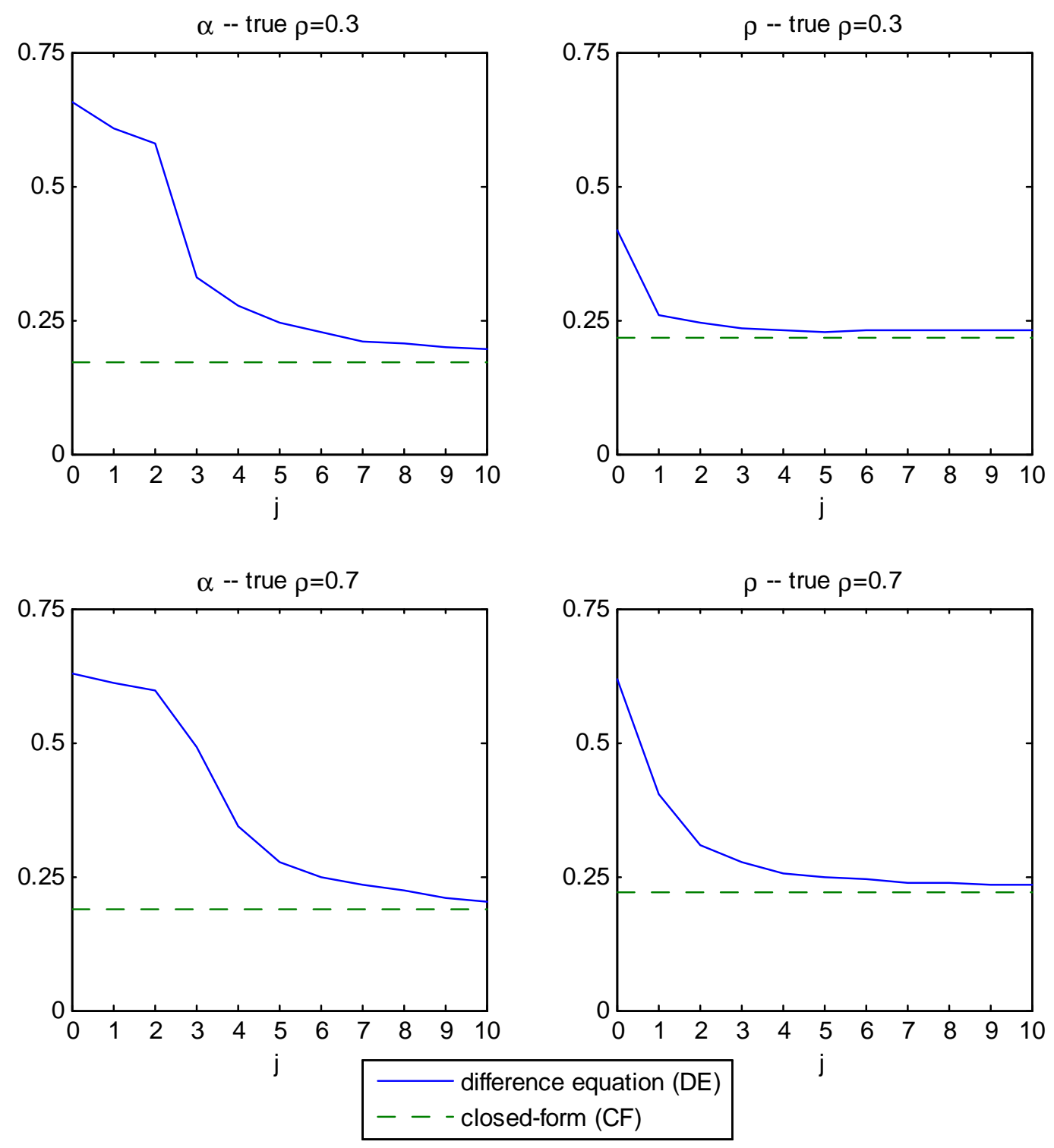

*The restrictions (constraints) are added to the DE specification.

Notes:

(1) $j$ is the number of additional restrictions;

(2) $j=0$ and $j \rightarrow \infty$ correspond to the DE and CF specifications, respectively;

(3) estimates are based on the data generating process with the real marginal cost follows an $\mathrm{AR}(2)$ process. 
Figure 4: Monte Carlo simulations, estimates of $\alpha$

Difference equation (DE) vs. closed-form $(\mathrm{CF})$

(Allowing for inflation feedback in the marginal cost equation)

DE
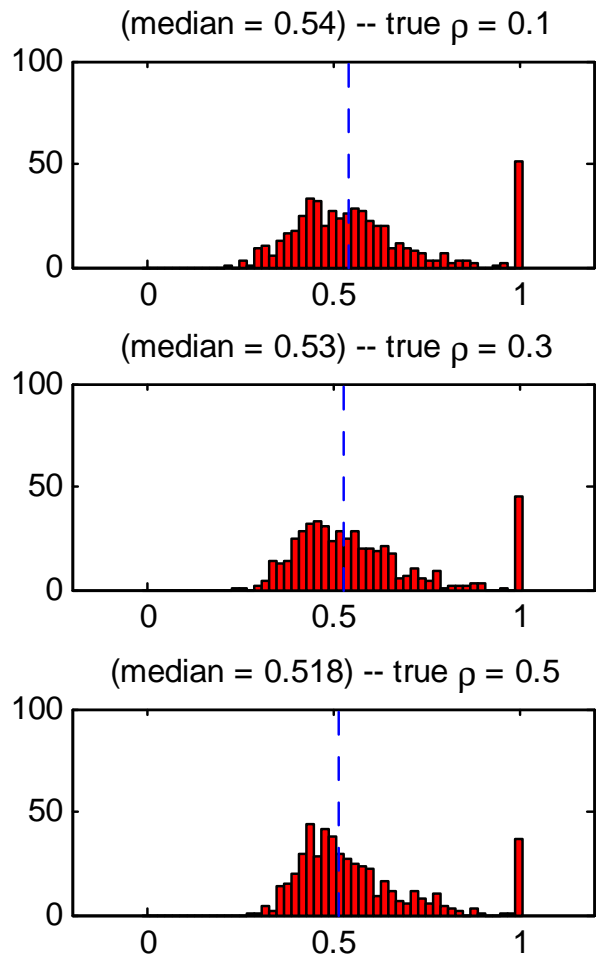

(median $=0.593$ ) -- true $\rho=0.7$

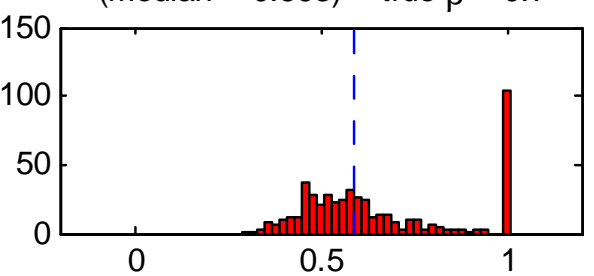

(median $=1)$-- true $\rho=0.9$

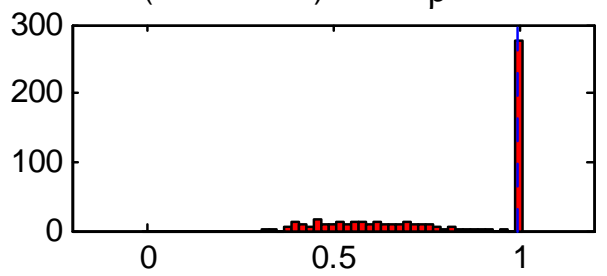

$\mathrm{CF}$

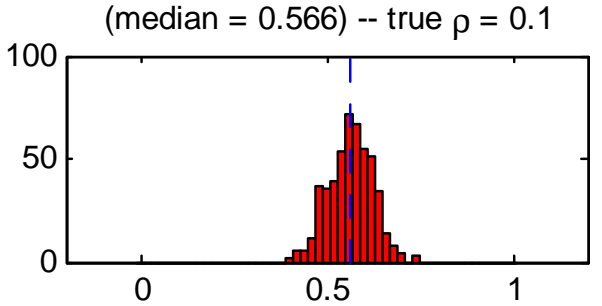

(median $=0.567)$-- true $\rho=0.3$

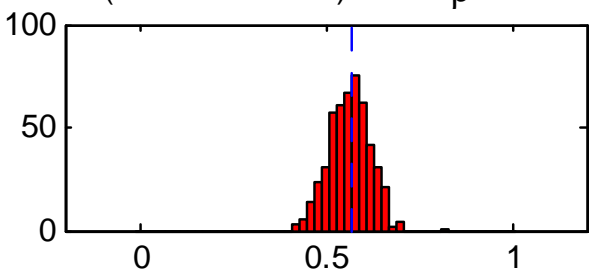

(median $=0.572)$-- true $\rho=0.5$

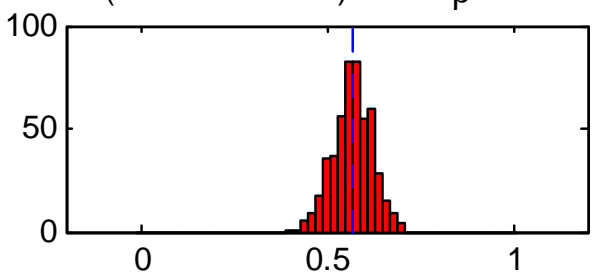

(median $=0.571)$-- true $\rho=0.7$

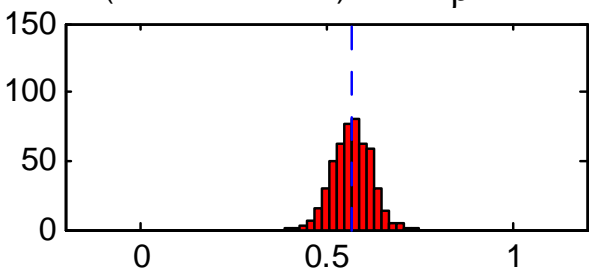

(median $=0.576)$-- true $\rho=0.9$

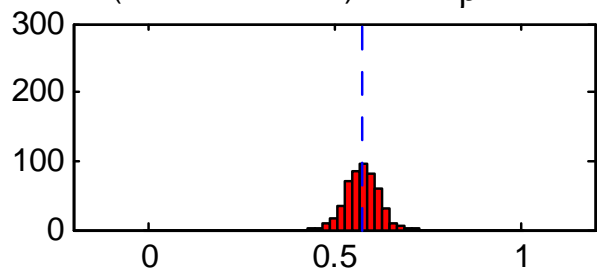

Notes:

(1) left and right panels correspond to DE and CF specifications, respectively;

(2) the vertical axis is the number of repetitions. Total number of repetitions is 500. 
Figure 5: Monte Carlo simulations, estimates of $\rho$

Difference equation (DE) vs. closed-form (CF)

(Allowing for inflation feedback in the marginal cost equation)

DE

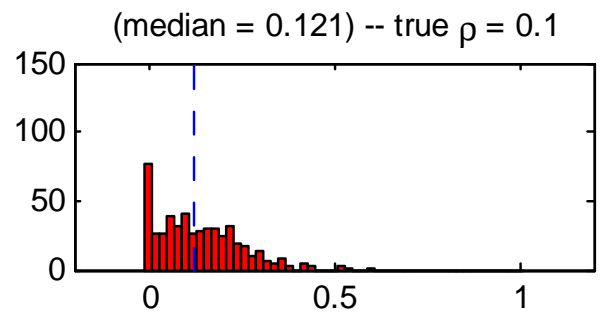

(median $=0.32$ ) - true $\rho=0.3$

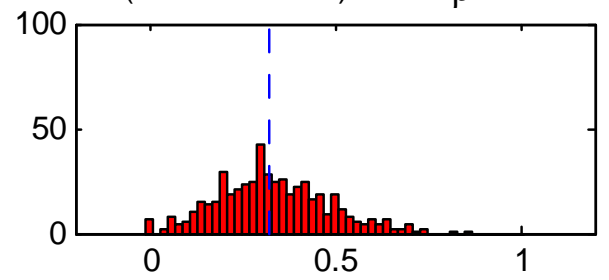

(median $=0.508)-$ true $\rho=0.5$
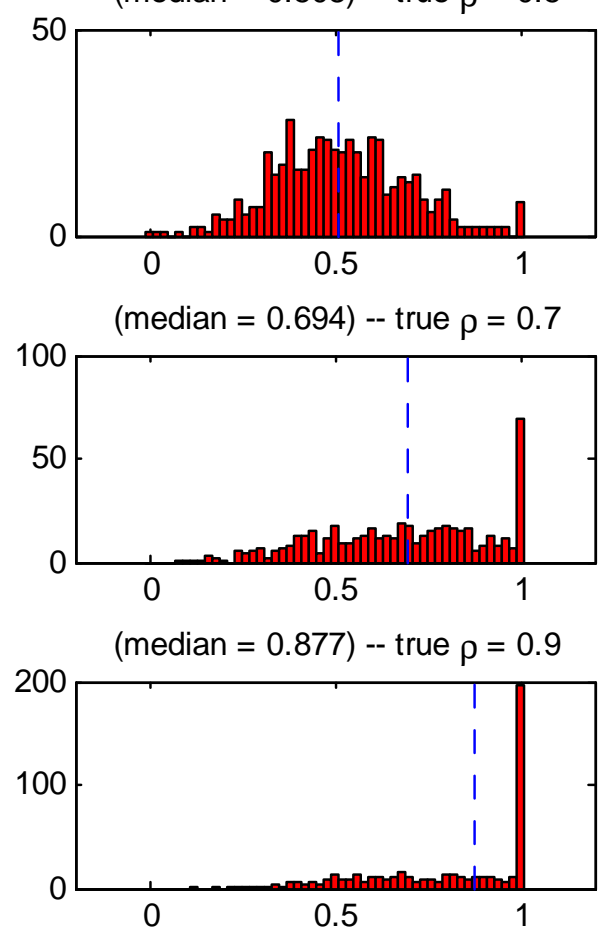

$\mathrm{CF}$

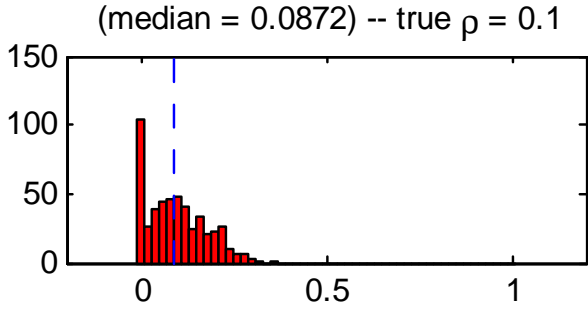

(median $=0.29)$-- true $\rho=0.3$

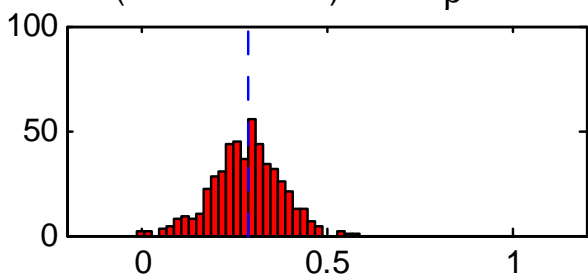

(median $=0.486)-$ true $\rho=0.5$
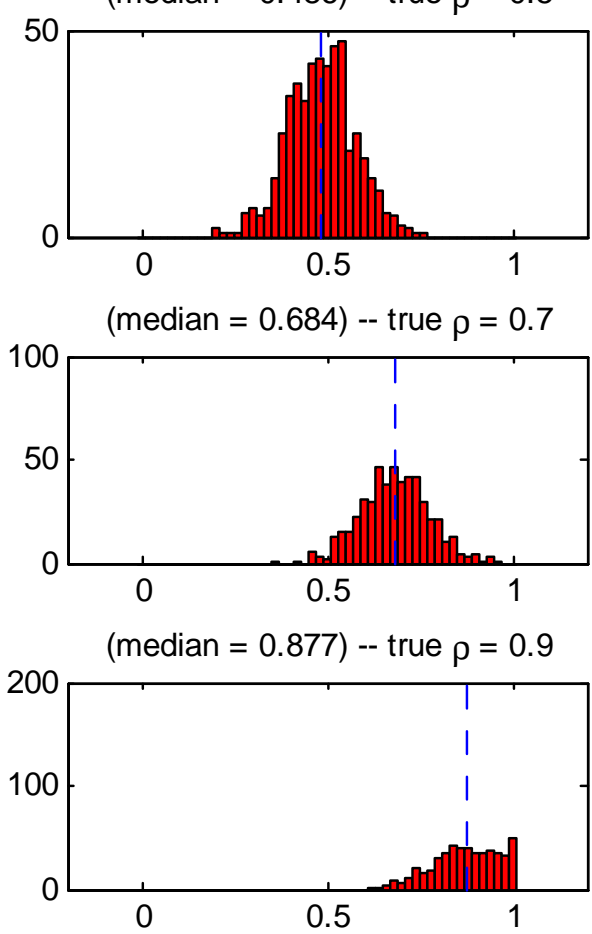

Notes:

(1) left and right panels correspond to DE and CF specifications, respectively;

(2) the vertical axis is the number of repetitions. Total number of repetitions is 500 . 
Figure 6: Monte Carlo simulations, estimates of $\alpha$ Difference equation (DE) vs. closed-form (CF)

(Larger information set)

DE

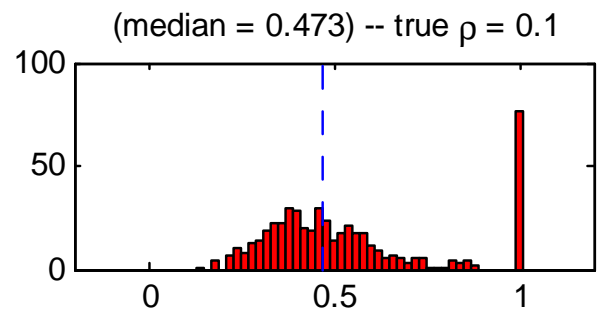

(median $=0.521)$-- true $\rho=0.3$

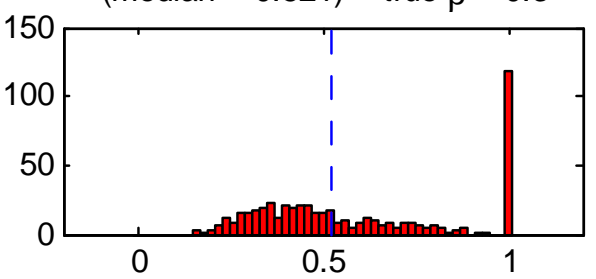

$($ median $=0.567)-$ true $\rho=0.5$

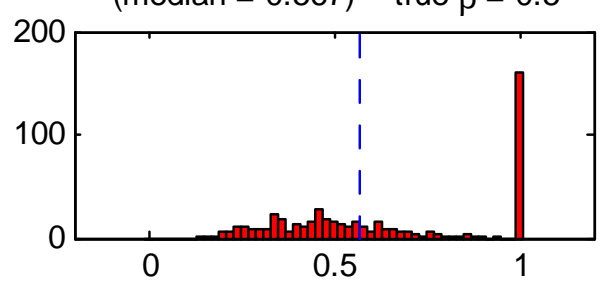

$($ median $=0.584)$-- true $\rho=0.7$

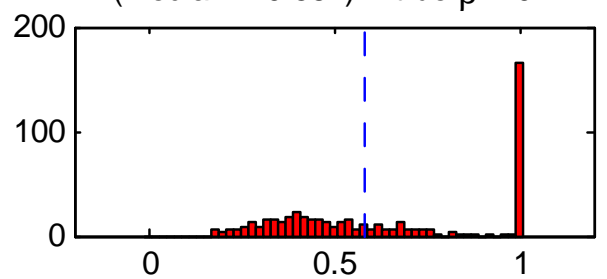

(median $=0.716)$-- true $\rho=0.9$

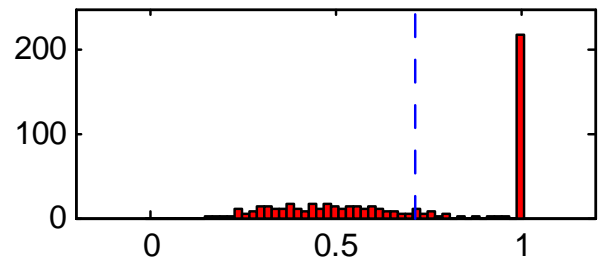

$\mathrm{CF}$

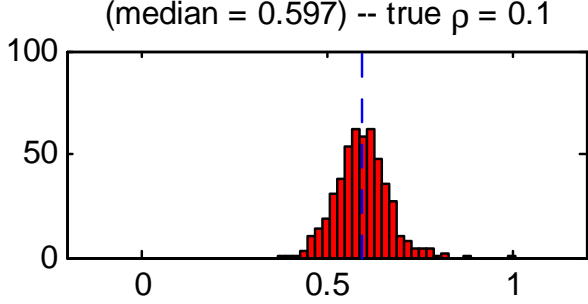

(median $=0.589)-$ true $\rho=0.3$

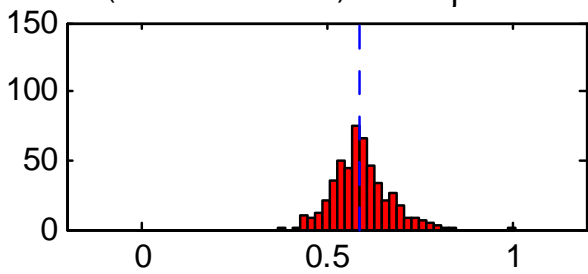

(median $=0.6)$-- true $\rho=0.5$

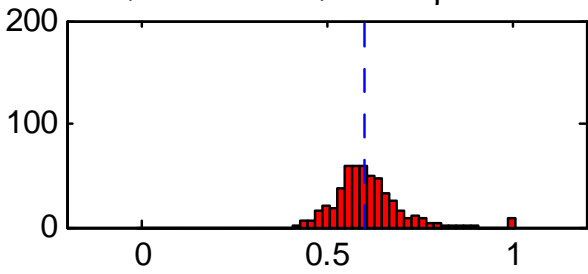

(median $=0.594)$-- true $\rho=0.7$

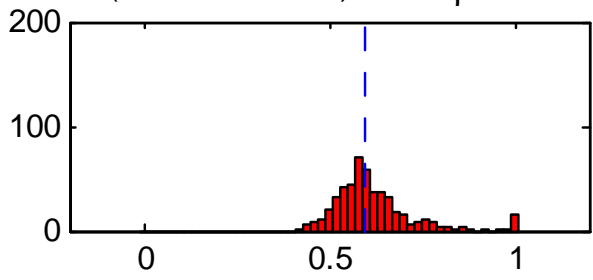

(median $=0.623$ ) - - true $\rho=0.9$

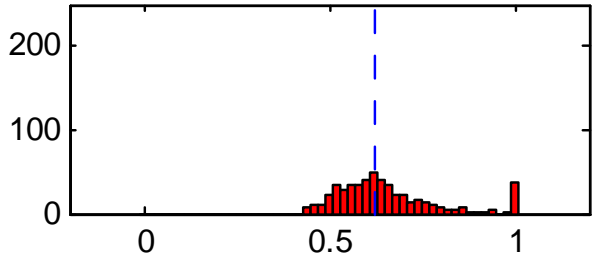

Notes:

(1) left and right panels correspond to DE and CF specifications, respectively;

(2) the vertical axis is the number of repetitions. Total number of repetitions is 500 . 
Figure 7: Monte Carlo simulations, estimates of $\rho$ Difference equation (DE) vs. closed-form (CF)

(Larger information set)

DE

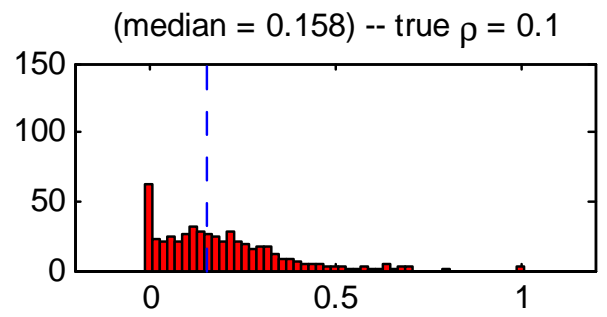

(median $=0.355)$-- true $\rho=0.3$

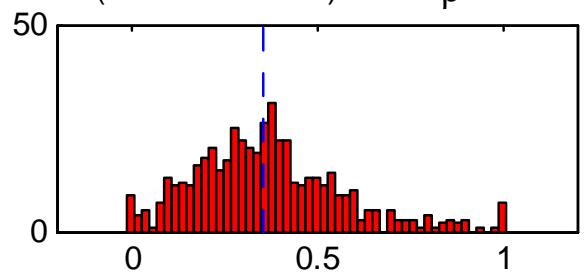

(median $=0.515)$-- true $\rho=0.5$

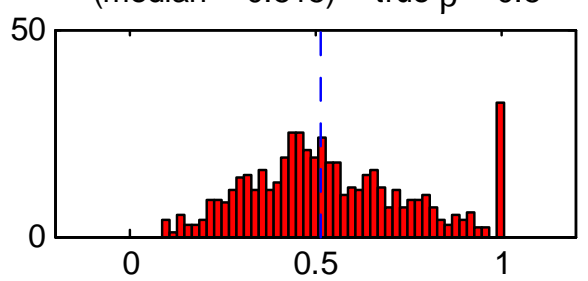

(median $=0.739)$-- true $\rho=0.7$
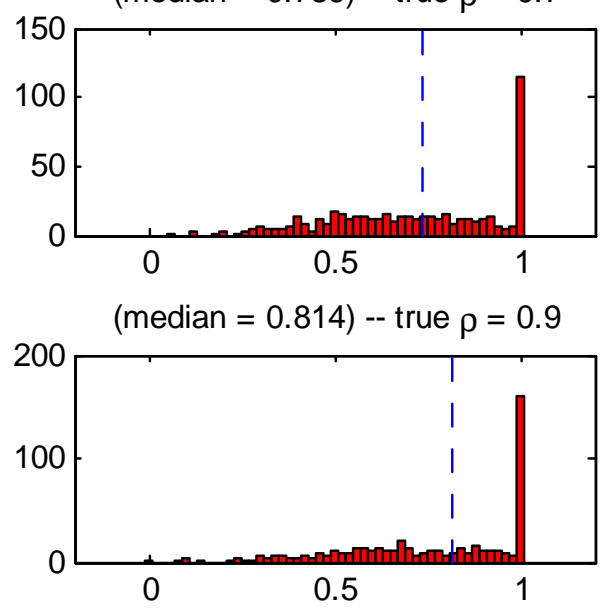

$\mathrm{CF}$

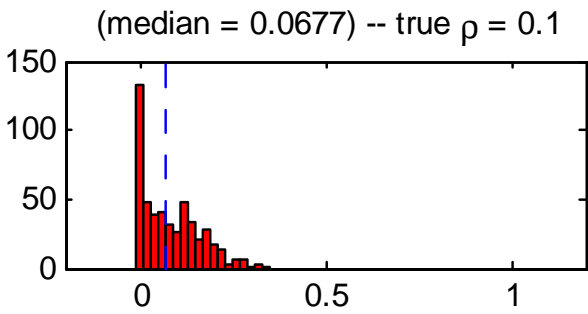

(median $=0.261$ ) - true $\rho=0.3$

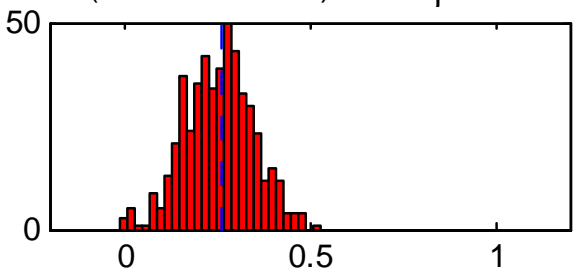

$($ median $=0.46)$-- true $\rho=0.5$

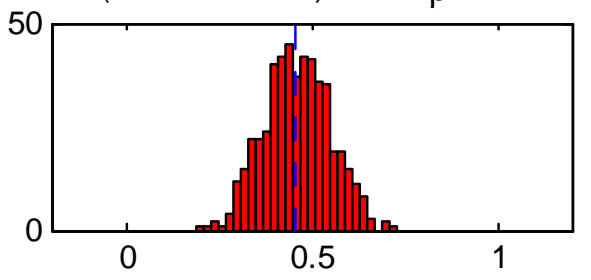

(median $=0.661)$-- true $\rho=0.7$
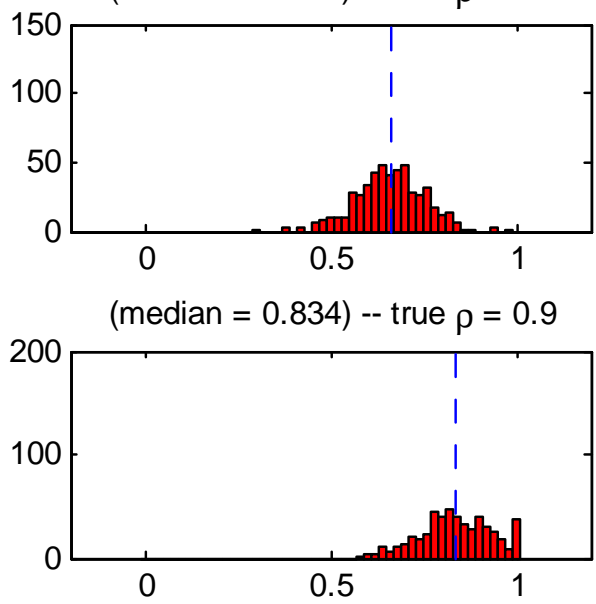

Notes:

(1) left and right panels correspond to DE and CF specifications, respectively;

(2) the vertical axis is the number of repetitions. Total number of repetitions is 500 . 
Figure 8: Monte Carlo simulations, $\alpha$ parameter

Difference equation (DE) vs. closed-form (CF), with misspecification*

DE

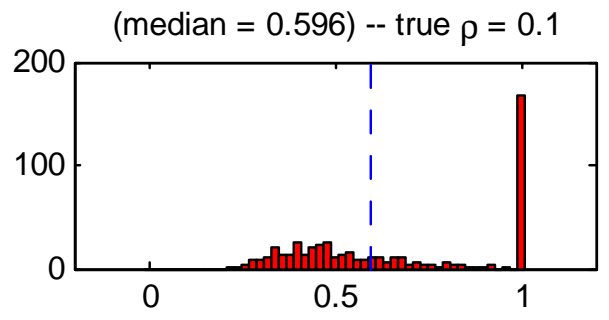

(median $=1)$-- true $\rho=0.3$

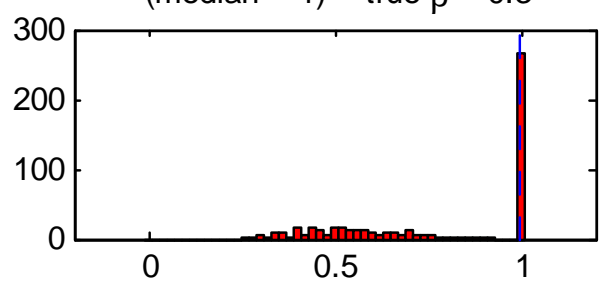

(median $=1$ ) - - true $\rho=0.5$

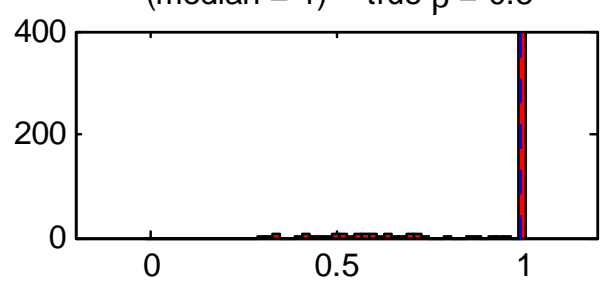

(median $=1$ ) -- true $\rho=0.7$

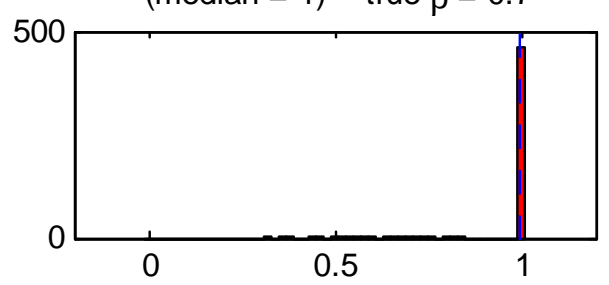

(median $=1$ ) -- true $\rho=0.9$

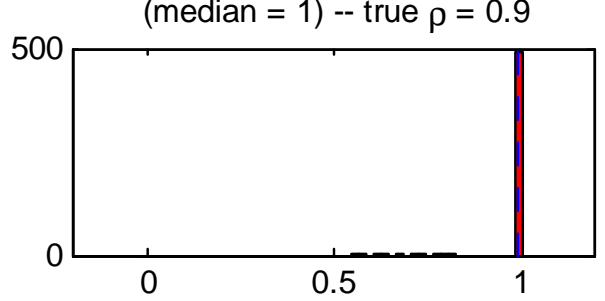

$\mathrm{CF}$
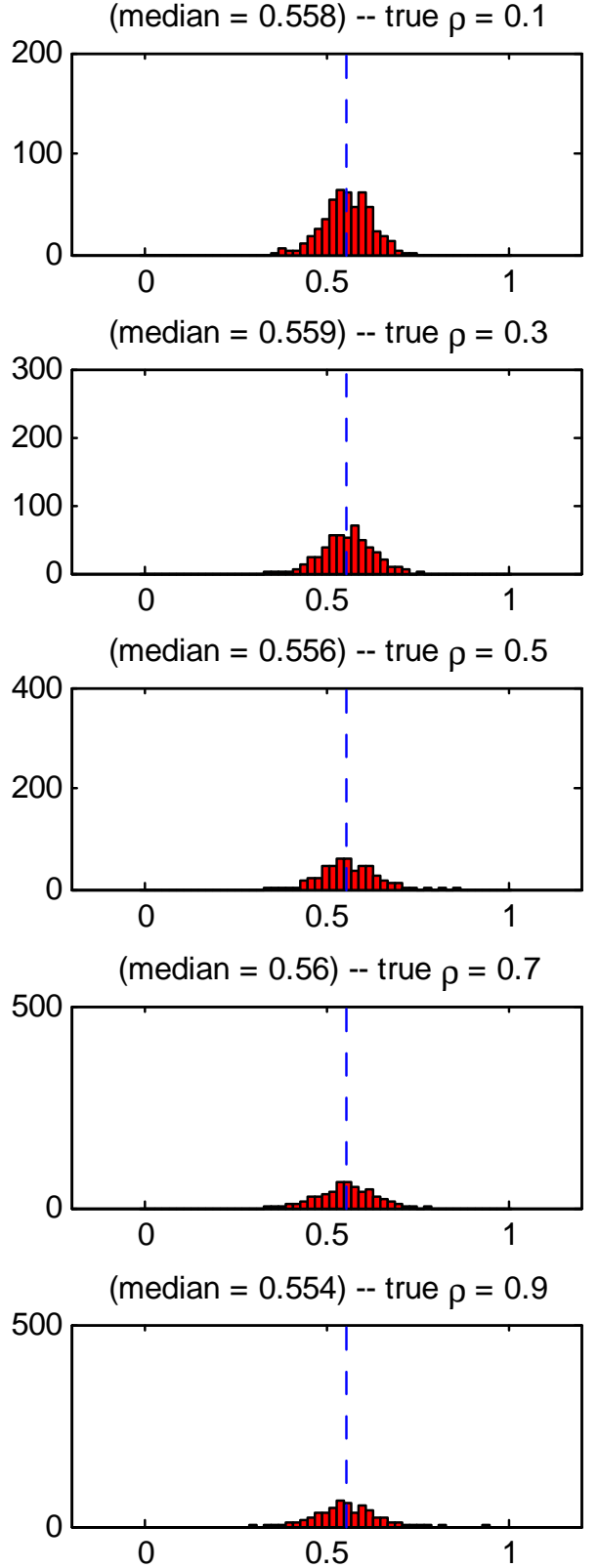

* The data are generated with $\tau=0.6$, but estimations are done with $\tau=1$.

Notes:

(1) left and right panels correspond to DE and CF specifications, respectively;

(2) the vertical axis is the number of repetitions. Total number of repetitions is 500. 
Figure 9: Monte Carlo simulations, $\rho$ parameter

Difference equation (DE) vs. closed-form (CF), with misspecification*

$\mathrm{DE}$
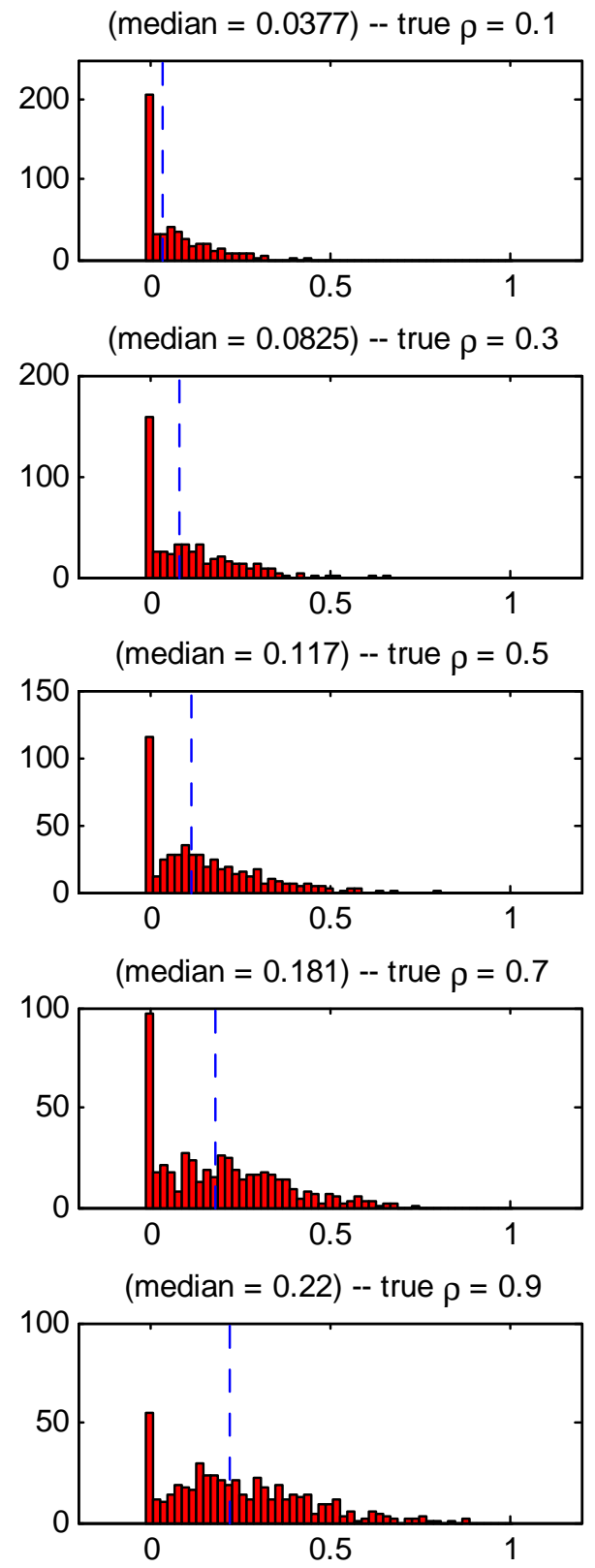

$\mathrm{CF}$
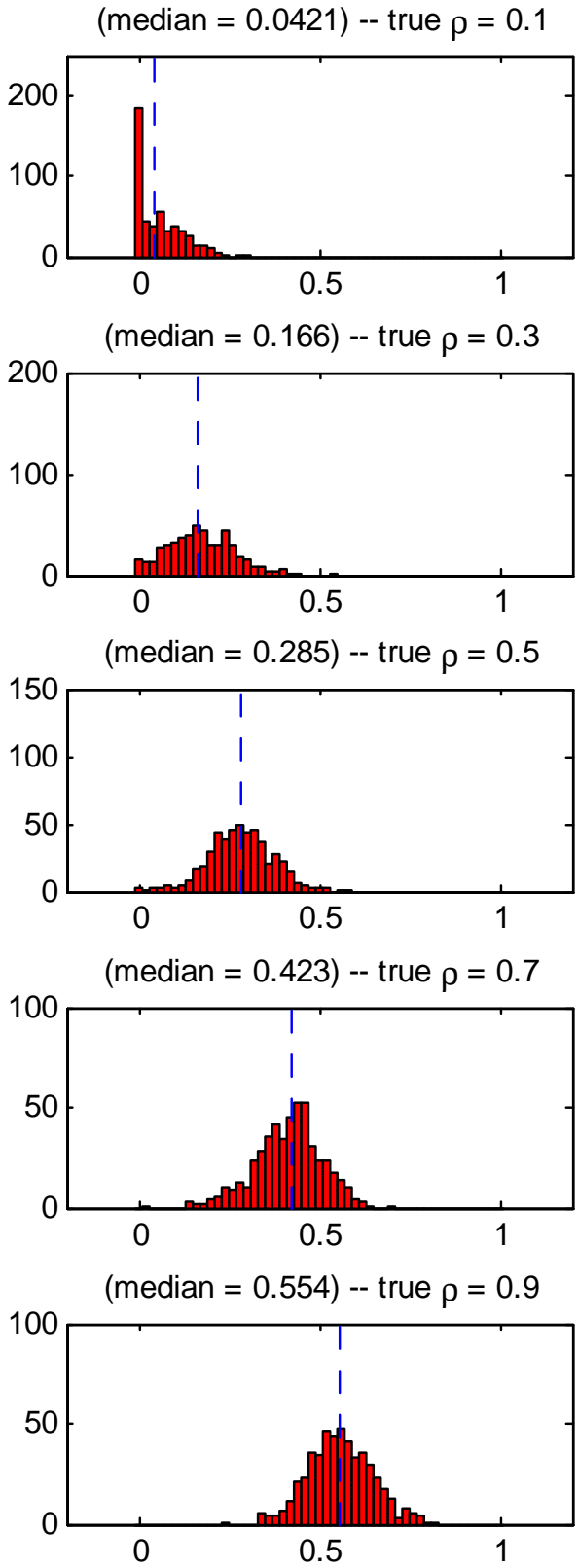

* The data are generated with $\tau=0.6$, but estimations are done with $\tau=1$. Notes:

(1) left and right panels correspond to DE and CF specifications, respectively;

(2) the vertical axis is the number of repetitions. Total number of repetitions is 500 . 
Figure 10: Parameter estimates after imposing additional expectations restrictions* Sample period: 1960:Q1-2003:Q4

(90\% and 99\% confidence intervals - unconstrained $\tau$ )

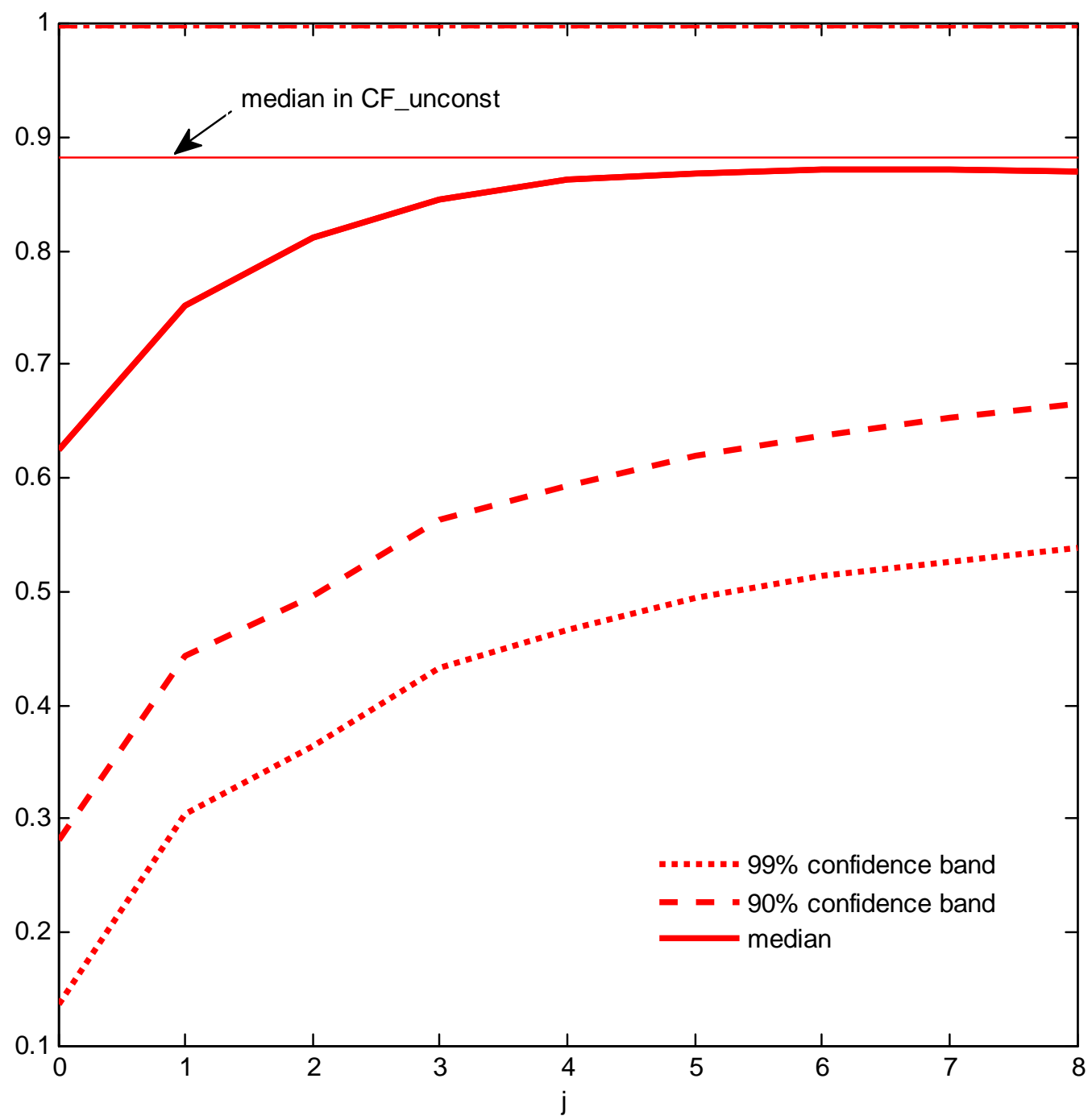

*The restrictions (constraints) are added to the DE specification.

Notes:

(1) $j$ is the number of additional restrictions;

(2) $j=0$ and $j \rightarrow \infty$ correspond to the $D E_{-}$unconst and $C F_{-}$unconst specifications, respectively. 
Table 1: Structural parameter estimates (median and 90\% confidence interval) Sample period: 1960:Q1-2003:Q4

\begin{tabular}{|l|c|c|c|c|}
\hline & $\rho$ & $\alpha$ & $\theta$ & $\tau$ \\
\hline \hline DE_const & 0 & 0.582 & 9.78 & 1 \\
& $(0,0.16)$ & $(0.45,0.67)$ & $(7.56,12.49)$ & - \\
\hline DE_unconst & 0.63 & 0.599 & 11.90 & 0.56 \\
& $(0.28,1)$ & $(0.51,0.72)$ & $(10.27,14.80)$ & $(0.22,0.80)$ \\
\hline CF_const & 0.51 & 0.797 & 11.21 & 1 \\
& $(0.25,0.91)$ & $(0.67,0.87)$ & $(8.04,81.67)$ & - \\
\hline CF_unconst & 0.88 & 0.863 & 12.58 & 0.68 \\
& $(0.75,0.99)$ & $(0.75,0.92)$ & $(10.86,94.97)$ & $(0.490 .89)$ \\
\hline
\end{tabular}

Notes: (1) numbers in parentheses are $90 \%$ confidence intervals; (2) DE_const and $C F$ _ const correspond to difference equation (DE) and closed-form (CF) specifications with $\tau=1$; (3) DE_ unconst and $C_{-}$unconst correspond to $\mathrm{DE}$ and $\mathrm{CF}$ cases with unconstrained $\tau$, respectively; (4) DE_const is the benchmark case in Cogley and Sbordone (2008).

Table 2: Structural parameter estimates (median and 90\% confidence interval)

Sample period: 1960:Q1-2003:Q4

(removing ensembles with $\theta>40$ )

\begin{tabular}{|l|c|c|c|c|}
\hline & $\rho$ & $\alpha$ & $\theta$ & $\tau$ \\
\hline \hline DE_const & 0 & 0.582 & 9.78 & 1 \\
& $(0,0.16)$ & $(0.45,0.67)$ & $(7.56,12.49)$ & - \\
\hline DE_unconst & 0.63 & 0.599 & 11.89 & 0.56 \\
& $(0.28,1)$ & $(0.51,0.72)$ & $(10.25,14.68)$ & $(0.22,0.80)$ \\
\hline CF_const & 0.49 & 0.804 & 10.76 & 1 \\
& $(0.24,0.78)$ & $(0.70,0.87)$ & $(7.98,25.62)$ & - \\
\hline CF_unconst & 0.87 & 0.868 & 12.38 & 0.68 \\
& $(0.75,0.98)$ & $(0.78,0.92)$ & $(10.81,28.82)$ & $(0.490 .89)$ \\
\hline
\end{tabular}

Notes: (1) numbers in parentheses are $90 \%$ confidence intervals; (2) DE_const and $C F_{-}$const correspond to difference equation (DE) and closed-form (CF) specifications with $\tau=1$; (3) DE_unconst and $C F$ unconst correspond to $\mathrm{DE}$ and $\mathrm{CF}$ cases with unconstrained $\tau$, respectively; (4) DE_const is the benchmark case in Cogley and Sbordone (2008). 


\section{Appendix A: Derivation of NKPC in difference equation (DE) form}

In this appendix, we derive the NKPC in difference-equation (DE) form as described in (32) and (33). We also show how to combine them into a final form used in the estimation procedure described in section 3.2. and show the cross-equation restrictions implied by conditional expectation based on information at $t-2$. The NKPC derivation closely follows that in Cogley and Sbordone (2008). ${ }^{30}$

First, let's derive the log-linear approximation of the evolution of aggregate prices. Let $X_{t}$ be the optimal nominal price at time $t$ chosen by firms that are allowed to adjust their prices (with probability $(1-\alpha)$ in a Calvo setup). Based on our indexation mechanism, the price of an individual firm $i$ that is not allowed to adjust (with probability $\alpha$ ) evolves according to

$$
P_{t}(i)=\left(\Pi_{t-1}^{\tau} \Pi_{t-2}^{1-\tau}\right)^{\rho} P_{t-1}(i)
$$

Hence, the aggregate price based on the CES aggregator is given by

$$
P_{t}=\left[(1-\alpha) X_{t}^{1-\theta}+\alpha\left\{\left(\Pi_{t-1}^{\tau} \Pi_{t-2}^{1-\tau}\right)^{\rho} P_{t-1}\right\}^{1-\theta}\right]^{\frac{1}{1-\theta}} .
$$

Dividing by the price level $P_{t}$, we have

$$
1=(1-\alpha) x_{t}^{1-\theta}+\alpha\left\{\left(\Pi_{t-1}^{\tau} \Pi_{t-2}^{1-\tau}\right)^{\rho} \Pi_{t}^{-1}\right\}^{1-\theta}
$$

where $x_{t}$ is the optimal relative price at time $t$. Next define stationary variables $\widetilde{\Pi}_{t}=\Pi_{t} / \bar{\Pi}_{t}$, $g_{t}^{\bar{\pi}}=\bar{\Pi}_{t} / \bar{\Pi}_{t-1}, g_{t}^{y}=Y_{t} / Y_{t-1}$, and $\widetilde{x}_{t}=x_{t} / \bar{x}_{t}$. Here, for any variable $k_{t}, \bar{k}_{t}$ is its time-varying trend. (A1) can then be transformed in terms of these stationary variables to yield (after some algebra):

$$
\begin{aligned}
1= & (1-\alpha) \widetilde{x}_{t}^{1-\theta} \bar{x}_{t}^{1-\theta} \\
& +\alpha\left[\begin{array}{c}
\widetilde{\Pi}_{t-2}^{\rho(1-\tau)(1-\theta)} \widetilde{\Pi}_{t-1}^{\rho \tau(1-\theta)} \widetilde{\Pi}_{t}^{-(1-\theta)} \bar{\Pi}_{t}^{(1-\rho)(\theta-1)} \\
\left(g_{t-1}^{\bar{\pi}}\right)^{-\rho(1-\tau)(1-\theta)}\left(g_{t}^{\bar{\pi}}\right)^{-\rho(1-\tau)(1-\theta)}\left(g_{t}^{\bar{\pi}}\right)^{-\rho \tau(1-\theta)}
\end{array}\right] .
\end{aligned}
$$

In the steady state where $\widetilde{x}_{t}=\widetilde{\Pi}_{t}=g_{t}^{\bar{\pi}}=1,(\mathrm{~A} 2)$ can be solved for $\bar{x}_{t}$ as a function of $\bar{\Pi}_{t}$ :

$$
\bar{x}_{t}=\left[\frac{1-\alpha \bar{\Pi}_{t}^{(1-\rho)(\theta-1)}}{1-\alpha}\right]^{\frac{1}{1-\theta}} .
$$

\footnotetext{
${ }^{30}$ Note that in Cogley and Sbordone (2008), the indexation is constrained to the first lag of inflation, i.e. $\tau=1$.
} 
Defining $\widehat{\pi}_{t} \equiv \ln \widetilde{\Pi}_{t} \equiv \ln \left(\Pi_{t} / \bar{\Pi}_{t}\right)$ and $\widehat{x}_{t} \equiv \ln \widetilde{x}_{t}$, imposing (A3), and rearranging, the log-linear approximation of (A2) around the steady state can be expressed as

$$
\begin{aligned}
\widehat{x}_{t}= & -\frac{1}{\varphi_{0, t}} \rho(1-\tau)\left(\widehat{\pi}_{t-2}-\widehat{g}_{t-1}^{\bar{\pi}}-\widehat{g}_{t}^{\bar{\pi}}\right) \\
& -\frac{1}{\varphi_{0, t}} \rho \tau\left(\widehat{\pi}_{t-1}-\widehat{g}_{t}^{\bar{\pi}}\right) \\
& +\frac{1}{\varphi_{0, t}} \widehat{\pi}_{t}
\end{aligned}
$$

where $\varphi_{0, t}=\frac{1-\alpha \bar{\Pi}_{t}^{(1-\rho)(\theta-1)}}{\alpha \bar{\Pi}_{t}^{(1-\rho)(\theta-1)}}$.

Next, we take the log-linear approximation to the first-order condition (FOC) of firms' pricing problem. Identically to the one-lag indexation case in Cogley and Sbordone (2008), the firms' FOC can be written as

$$
E_{t} \sum_{j=0}^{\infty} \alpha^{j} Q_{t, t+j} Y_{t+j} P_{t+j} \Psi_{t j}^{1-\theta}\left(X_{t}^{(1+\theta \omega)}-\frac{\theta}{\theta-1} M C_{t+j} \Psi_{t j}^{-(1+\theta \omega)} P_{t+j}^{\theta \omega}\right)=0
$$

where $Q_{t, t+j}$ and $M C_{t+j}$ are the nominal discount factor and average marginal cost at $t+j$, respectively. The variable $\Psi_{t j}$ enters in the CES demand function for any good i, $Y_{t+j}(i)=$ $Y_{t+j}\left(\frac{P_{t+j}(i) \Psi_{t j}}{P_{t+j}}\right)$, with

$$
\Psi_{t j}=\left\{\begin{array}{cl}
1 & j=0 \\
\prod_{k=0}^{j-1}\left(\Pi_{t+k}^{\tau} \Pi_{t+k-1}^{1-\tau}\right)^{\rho} & j \geq 1
\end{array}\right.
$$

The second line of (A5) makes clear that prices are indexed to a weighted average of the first two lags of inflation if they are not set optimally. Combining (A4) and (A5) and rearranging leads to

$$
X_{t}^{1+\theta \omega}=\frac{C_{t}}{D_{t}}
$$

where $C_{t}$ and $D_{t}$ are recursively defined by

$$
\begin{aligned}
C_{t}= & \frac{\theta}{\theta-1} Y_{t} P_{t}^{\theta(1+\omega)-1} M C_{t} \\
& +E_{t}\left[\alpha q_{t, t+1} \Pi_{t}^{-\rho \tau \theta(1+\omega)} \Pi_{t-1}^{-\rho(1-\tau) \theta(1+\omega)} C_{t+1}\right] \\
D_{t}= & Y_{t} P_{t}^{\theta-1} \\
& +E_{t}\left[\alpha q_{t, t+1} \Pi_{t}^{\rho \tau(1-\theta)} \Pi_{t-1}^{\rho(1-\tau)(1-\theta)} D_{t+1}\right]
\end{aligned}
$$


where $q_{t, t+1}$ now is the real discount factor. Defining the stationary variables $\widetilde{C}_{t}=\frac{C_{t}}{Y_{t} P_{t}^{\theta(1+\omega)}}$ and $\widetilde{D}_{t}=\frac{D_{t}}{Y_{t} P_{t}^{\theta-1}}$, we have based on (A6) and (A7):

$$
\begin{aligned}
\widetilde{C}_{t}= & \frac{\theta}{\theta-1} m c_{t} \\
& +E_{t}\left[\alpha q_{t, t+1} g_{t+1}^{y} \Pi_{t+1}^{\theta(1+\omega)} \Pi_{t}^{-\rho \tau \theta(1+\omega)} \Pi_{t-1}^{-\rho(1-\tau) \theta(1+\omega)} \widetilde{C}_{t+1}\right] \\
\widetilde{D}_{t}= & 1+E_{t}\left[\alpha q_{t, t+1} g_{t+1}^{y} \Pi_{t+1}^{(\theta-1)} \Pi_{t}^{\rho \tau(1-\theta)} \Pi_{t-1}^{\rho(1-\tau)(1-\theta)} \widetilde{D}_{t+1}\right] .
\end{aligned}
$$

Also note that

$$
\frac{\widetilde{C}_{t}}{\widetilde{D}_{t}}=\frac{C_{t}}{D_{t}} \frac{1}{P_{t}^{(1+\theta \omega)}}=x_{t}^{1+\theta \omega},
$$

where $x_{t} \equiv X_{t} / P_{t}$. Evaluating (A8) and (A9) at the steady state leads to

$$
\begin{aligned}
& \bar{C}_{t}=\frac{\frac{\theta}{\theta-1} \overline{m c}_{t}}{1-\alpha \overline{q g}^{y} \bar{\Pi}_{t}^{\theta(1+\omega)(1-\rho)}} \\
& \bar{D}_{t}=\frac{1}{1-\alpha \overline{q g}^{y} \bar{\Pi}_{t}^{(\theta-1)(1-\rho)}}
\end{aligned}
$$

Combining the two expressions above with (A3) and using (A10) leads to the steady-state restriction (31). This restriction does not depend on $\tau$ and hence is identical to the case in Cogley and Sbordone (2008) with $\tau=1$. Next, define $\widehat{C}_{t}=\ln \frac{\widetilde{C}_{t}}{\bar{C}_{t}}, \widehat{D}_{t}=\ln \frac{\widetilde{D}_{t}}{\bar{D}_{t}}$, and $\widehat{m c}_{t}=\ln \frac{m c_{t}}{m c_{t}}$. Log-linearizing (A10) yields

$$
(1+\theta \omega) \widehat{x}_{t}=\left(\widehat{C}_{t}-\widehat{D}_{t}\right)
$$

Combining (A11) with (A3) and rearranging leads to an intermediate expression for $\widehat{\pi}_{t}$ :

$$
\begin{aligned}
\widehat{\pi}_{t}= & \rho \tau\left[\widehat{\pi}_{t-1}-\widehat{g}_{t}^{\bar{\pi}}\right] \\
& +\rho(1-\tau)\left[\widehat{\pi}_{t-2}-\widehat{g}_{t-1}^{\bar{\pi}}-\widehat{g}_{t}^{\bar{\pi}}\right] \\
& +\frac{\varphi_{0, t}}{(1+\theta \omega)}\left(\widehat{C}_{t}-\widehat{D}_{t}\right) .
\end{aligned}
$$

We can obtain the expressions for $\widehat{C}_{t}$ and $\widehat{D}_{t}$ by log-linearizing (A8) and (A9). Combining the resulting expressions with (A11) leads to equations (32) and (33) in the main text:

$$
\begin{aligned}
\widehat{\pi}_{t}= & \rho \tau\left(\widehat{\pi}_{t-1}-\widehat{g}_{t}^{\bar{\pi}}\right)+\rho(1-\tau)\left(\widehat{\pi}_{t-2}-\widehat{g}_{t-1}^{\bar{\pi}}-\widehat{g}_{t}^{\bar{\pi}}\right) \\
& +\lambda_{t} E_{t}\left(\widehat{\pi}_{t+1}-\rho \tau \widehat{\pi}_{t}-\rho(1-\tau)\left(\widehat{\pi}_{t-1}-\widehat{g}_{t}^{\bar{\pi}}\right)\right)+\zeta_{t} \widehat{m c}_{t}+\gamma_{t} \widehat{D}_{t} \\
\widehat{D}_{t}= & \varphi_{1 t} E_{t}\left(\widehat{q}_{t, t+1}+\widehat{g}_{t+1}^{y}\right) \\
& +\varphi_{1 t}(\theta-1) E_{t}\left\{\widehat{\pi}_{t+1}-\rho \tau \widehat{\pi}_{t}-\rho(1-\tau)\left(\widehat{\pi}_{t-1}-\widehat{g}_{t}^{\bar{\pi}}\right)\right\}+\varphi_{1 t} E_{t} \widehat{D}_{t+1},
\end{aligned}
$$


with the time-varying coefficients given by

$$
\begin{aligned}
\zeta_{t} & =\chi_{t} \varphi_{3 t} \\
\lambda_{t} & =\varphi_{2 t}\left(1+\varphi_{0 t}\right) \\
\gamma_{t} & =\frac{\chi_{t}\left(\varphi_{2 t}-\varphi_{1 t}\right)}{\varphi_{1 t}} \\
\chi_{t} & =\frac{\varphi_{0 t}}{1+\theta \omega} \\
\varphi_{1 t} & =\alpha \overline{q g} y \bar{\Pi}_{t}^{(\theta-1)(1-\rho)} \\
\varphi_{2 t} & =\alpha \overline{q g} y \bar{\Pi}_{t}^{\theta(1+\omega)(1-\rho)} \\
\varphi_{3 t} & =1-\varphi_{2 t}
\end{aligned}
$$

Finally, iterating $\widehat{D}_{t}$ in (A14) forward, substituting the resulting expression for $\widehat{D}_{t}$ in (A13), converting real discount factor $\widehat{q}_{t, t+j}$ into nominal discount factor $\widetilde{Q}_{t, t+j}$, and rearranging lead to the NKPC in DE form:

$$
\begin{aligned}
\widehat{\pi}_{t}= & \widetilde{\rho}_{1 t}^{D}\left(\widehat{\pi}_{t-1}-\widehat{g}_{t}^{\bar{\pi}}\right)+(1-\tau) \widetilde{\rho}_{2 t}^{D}\left(\widehat{\pi}_{t-2}-\widehat{g}_{t-1}^{\bar{\pi}}-\widehat{g}_{t}^{\bar{\pi}}\right) \\
& +\widetilde{\zeta}_{t}^{D} \widehat{m c}_{t} \\
& +d_{1 t}^{D} E_{t} \widehat{\pi}_{t+1} \\
& +d_{2 t}^{D} E_{t} \sum_{j=2}^{\infty} \varphi_{1 t}^{j-1} \widehat{\pi}_{t+j} \\
& +d_{3 t}^{D} E_{t} \sum_{j=0}^{\infty} \varphi_{1 t}^{j}\left[\widehat{Q}_{t+j, t+j+1}+\widehat{g}_{t+j+1}^{y}\right]
\end{aligned}
$$

where the coefficients are defined by

$$
\begin{aligned}
\widetilde{\rho}_{1 t}^{D} & =\left[\rho \tau-\lambda_{t} \rho(1-\tau)-\gamma_{t}(\theta-1) \rho(1-\tau) \varphi_{1 t}\right] / \Delta_{t} \\
\tilde{\rho}_{2 t}^{D} & =\rho / \Delta_{t} \\
d_{1 t}^{D} & =\widetilde{d}_{1 t}^{D}+d_{3 t}^{D} \\
d_{2 t}^{D} & =\widetilde{d}_{2 t}^{D}+d_{3 t}^{D} \\
d_{3 t}^{D} & =\left[\gamma_{t} \varphi_{1 t}\right] / \Delta_{t} \\
\widetilde{\zeta}_{t}^{D} & =\zeta_{t} / \Delta_{t} \\
\Delta_{t} & =1+\rho \tau \lambda_{t}+\gamma_{t}(\theta-1) \rho \varphi_{1 t}\left\{\tau+(1-\tau) \varphi_{1 t}\right\} \\
\widetilde{d}_{1 t}^{D} & =\left[\lambda_{t}+\gamma_{t}(\theta-1) \varphi_{1 t}\left\{1-\rho \tau \varphi_{1 t}-\rho(1-\tau) \varphi_{1 t}^{2}\right\}\right] / \Delta_{t} \\
\widetilde{d}_{2 t}^{D} & =\left[\gamma_{t}(\theta-1) \varphi_{1 t}\left\{1-\rho \tau \varphi_{1 t}-\rho(1-\tau) \varphi_{1 t}^{2}\right\}\right] / \Delta_{t}
\end{aligned}
$$


Note that as in Cogley and Sbordone (2008), we use the "anticipated utility" assumption (Kreps, $1998)$ in deriving the NKPC in (PC-DE) so that $E_{t} \prod_{k=0}^{i} \varphi_{1 t+k} \widehat{h}_{t+i}=\varphi_{1 t}^{i+1} E_{t} \widehat{h}_{t+i}$ for any variable $\widehat{h}_{t+i}$.

We note that there are two relevant limiting cases of (PC-DE). First, when $\tau=1$ so that the indexation is constrained to the first lag of inflation, we have the NKPC in Cogley and Sbordone (2008). Second, if the prices of non-adjusting firms are fully indexed to a mixture of past inflation (first and second lags) and current trend inflation, the NKPC collapses to the case with constant coefficients and where there is no extra lead terms beyond $t+1$. Furthermore, under constant trend case and when $\tau=1$, one obtains the NKPC as in Christiano, Eichenbaum, and Evans (2005) exhibited in (2).

Cross-equation restrictions Given the forecasting rule (36) and equation (PC-DE), we obtain the conditional expectation of inflation based on information at $t-2$ in the DE form as follows

$$
\begin{aligned}
\mathbf{e}_{\pi}^{\prime} \mathbf{A}_{t-2}^{2} \widehat{\mathbf{z}}_{t-2}= & \widetilde{\rho}_{1, t-2}^{D} \mathbf{e}_{\pi}^{\prime} \mathbf{A}_{t-2} \widehat{\mathbf{z}}_{t-2}+(1-\tau) \widetilde{\rho}_{2, t-2}^{D} \mathbf{e}_{\pi}^{\prime} \widehat{\mathbf{z}}_{t-2}+\widetilde{\zeta}_{t-2}^{D} \mathbf{e}_{m c}^{\prime} \mathbf{A}_{t-2}^{2} \widehat{\mathbf{z}}_{t-2} \\
& +d_{1 t-2}^{D} \mathbf{e}_{\pi}^{\prime} \mathbf{A}_{t-2}^{3} \widehat{\mathbf{z}}_{t-2}+d_{2 t-2}^{D} \varphi_{1 t-2} \mathbf{e}_{\pi}^{\prime} \mathbf{J}_{t-2} \mathbf{A}_{t-2}^{4} \widehat{\mathbf{z}}_{t-2} \\
& +d_{3 t-2}^{D}\left(\mathbf{e}_{Q}^{\prime} \mathbf{J}_{t-2} \mathbf{A}_{t-2}^{2} \widehat{\mathbf{z}}_{t-2}+\mathbf{e}_{g^{y}}^{\prime} \mathbf{J}_{t-2} \mathbf{A}_{t-2}^{3} \widehat{\mathbf{z}}_{t-2}\right),
\end{aligned}
$$

where $\mathbf{J}_{t} \equiv\left(\mathbf{I}-\varphi_{1 t} \mathbf{A}_{t}\right)^{-1}$. Hence, the vector of cross-equation restrictions is given by

$$
\begin{aligned}
\mathbf{e}_{\pi}^{\prime} \mathbf{A}_{t-2}^{2}= & \widetilde{\rho}_{1, t-2}^{D} \mathbf{e}_{\pi}^{\prime} \mathbf{A}_{t-2}+(1-\tau) \widetilde{\rho}_{2, t-2}^{D} \mathbf{e}_{\pi}^{\prime} \mathbf{I}+\widetilde{\zeta}_{t-2}^{D} \mathbf{e}_{m c}^{\prime} \mathbf{A}_{t-2}^{2} \\
& +d_{1 t-2}^{D} \mathbf{e}_{\pi}^{\prime} \mathbf{A}_{t-2}^{3}+d_{2 t-2}^{D} \varphi_{1 t-2} \mathbf{e}_{\pi}^{\prime} \mathbf{J}_{t-2} \mathbf{A}_{t-2}^{4} \\
& +d_{3 t-2}^{D}\left(\mathbf{e}_{Q}^{\prime} \mathbf{J}_{t-2} \mathbf{A}_{t-2}^{2}+\mathbf{e}_{g^{y}}^{\prime} \mathbf{J}_{t-2} \mathbf{A}_{t-2}^{3}\right) \\
\equiv & \mathbf{g}^{D}\left(\boldsymbol{\mu}_{t-2}, \mathbf{A}_{t-2}, \boldsymbol{\psi}\right)
\end{aligned}
$$

\section{Appendix B: Derivation of closed-form (CF) NKPC}

We derive the "closed-form" (CF) representation of NKPC based on (A13) and (A14) in appendix A.

First, define an auxiliary variable

$$
\widehat{B}_{t}=\widehat{\pi}_{t}-\rho \tau\left(\widehat{\pi}_{t-1}-\widehat{g}_{t}^{\bar{\pi}}\right)-\rho(1-\tau)\left(\widehat{\pi}_{t-2}-\widehat{g}_{t-1}^{\bar{\pi}}-\widehat{g}_{t}^{\bar{\pi}}\right),
$$


so that

$$
E_{t} \widehat{B}_{t+1}=\widehat{\pi}_{t+1}-\rho \tau \widehat{\pi}_{t}-\rho(1-\tau)\left(\widehat{\pi}_{t-1}-\widehat{g}_{t}^{\bar{\pi}}\right)
$$

Note that the expectation above reflects the fact that $\widehat{g}_{t}^{\bar{\pi}}$ is an innovation process so that $E_{t} \widehat{g}_{t+j}^{\bar{\pi}}=0$ for $j \geq 1$. Using this definition, we can rewrite (A13) as

$$
\widehat{B}_{t}=\lambda_{t} E_{t} \widehat{B}_{t+1}+\zeta_{t} \widehat{m c}_{t}+\gamma_{t} \widehat{D}_{t}
$$

Solving forward (B1) yields

$$
\widehat{B}_{t}=\zeta_{t} E_{t} \sum_{j=0}^{\infty} \lambda_{t}^{j} \widehat{m c}_{t+j}+\gamma_{t} E_{t} \sum_{j=0}^{\infty} \lambda_{t}^{j} \widehat{D}_{t+j}
$$

In deriving (B2) (and (B3) below), the "anticipated utility" assumption is used so that $E_{t} \zeta_{t+j} \prod_{k=0}^{j} \lambda_{t+k} \widehat{m c}_{t+j}=$ $\zeta_{t} \lambda_{t}^{j+1} E_{t} \widehat{m c}_{t+j}$ and $E_{t} \gamma_{t+j} \prod_{k=0}^{j} \lambda_{t+k} \widehat{D}_{t+j}=\gamma_{t} \lambda_{t}^{j+1} E_{t} \widehat{D}_{t+j}$ for any $j>0$. Next, solving forward (A14), converting real discount factors into nominal ones, and rearranging lead to

$$
\begin{aligned}
\widehat{D}_{t}= & \varphi_{1 t} E_{t} \sum_{j=0}^{\infty} \varphi_{1 t}^{j}\left[\widehat{Q}_{t+j, t+j+1}+\widehat{g}_{t+j+1}^{y}\right] \\
& -\kappa_{1 t}\left[\widehat{\pi}_{t-1}-\widehat{g}_{t}^{\bar{\pi}}\right]+\kappa_{2 t} \widehat{\pi}_{t}+\kappa_{3 t} \widehat{\pi}_{t+1} \\
& +\kappa_{3 t} E_{t} \sum_{j=2}^{\infty} \varphi_{1 t}^{j-1} \widehat{\pi}_{t+j},
\end{aligned}
$$

with the new coefficients defined by

$$
\begin{aligned}
& \kappa_{1 t}=(\theta-1) \rho(1-\tau) \varphi_{1 t} \\
& \kappa_{2 t}=(\theta-1) \rho \tau \varphi_{1 t}+(\theta-1) \rho(1-\tau) \varphi_{1 t}^{2} \\
& \kappa_{2 t}=\theta \varphi_{1 t}-(\theta-1) \rho \tau \varphi_{1 t}^{2}-(\theta-1) \rho(1-\tau) \varphi_{1 t}^{3}
\end{aligned}
$$

We next remove the auxiliary variables $\widehat{B}_{t}$ and $\widehat{D}_{t}$ and derive the NKPC. Using the definition of $\widehat{B}_{t}$, we reintroduce inflation into (B2) so that

$$
\begin{aligned}
\widehat{\pi}_{t}= & \rho \tau\left(\widehat{\pi}_{t-1}-\widehat{g}_{t}^{\bar{\pi}}\right)+\rho(1-\tau)\left(\widehat{\pi}_{t-2}-\widehat{g}_{t-1}^{\bar{\pi}}-\widehat{g}_{t}^{\bar{\pi}}\right) \\
& +\zeta_{t} E_{t} \sum_{j=0}^{\infty} \lambda_{t}^{j} \widehat{m c}_{t+j}+\gamma_{t} E_{t} \sum_{j=0}^{\infty} \lambda_{t}^{j} \widehat{D}_{t+j}
\end{aligned}
$$


Finally, we substitute for $\widehat{D}_{t+j}$ terms in (B4) using (B3) and rearrange the resulting expression to obtain the CF representation of NKPC:

$$
\begin{aligned}
\widehat{\pi}_{t}= & \widetilde{\rho}_{1 t}^{C}\left(\widehat{\pi}_{t-1}-\widehat{g}_{t}^{\bar{\pi}}\right)+(1-\tau) \widetilde{\rho}_{2 t}^{C}\left(\widehat{\pi}_{t-2}-\widehat{g}_{t-1}^{\bar{\pi}}-\widehat{g}_{t}^{\bar{\pi}}\right) \\
& +\widetilde{\zeta}_{t}^{C} E_{t} \sum_{j=0}^{\infty} \lambda_{t}^{j} \widehat{m c}_{t+j} \\
& +d_{0 t}^{C} E_{t} \sum_{k=0}^{\infty} \lambda_{t}^{k}\left[\widehat{\pi}_{t+k-1}-\widehat{g}_{t+k}^{\bar{\pi}}\right] \\
& +d_{1 t}^{C} E_{t} \sum_{k=0}^{\infty} \lambda_{t}^{k} \widehat{\pi}_{t+k} \\
& +d_{2 t}^{C} E_{t} \sum_{k=0}^{\infty} \lambda_{t}^{k} \widehat{\pi}_{t+k+1} \\
& +d_{2 t}^{C} E_{t} \sum_{k=0}^{\infty} \lambda_{t}^{k} \sum_{j=2}^{\infty} \varphi_{1 t}^{j-1} \widehat{\pi}_{t+j+k} \\
& +d_{3 t}^{C} E_{t} \sum_{k=0}^{\infty} \lambda_{t}^{k} \sum_{j=0}^{\infty} \varphi_{1 t}^{j}\left[\widehat{Q}_{t+j+k, t+j+k+1}+\widehat{g}_{t+j+k+1}^{y}\right]
\end{aligned}
$$

with the new coefficients defined as follows

$$
\begin{aligned}
& \widetilde{\rho}_{2 t}^{C}=\rho \tau \\
& \widetilde{\rho}_{2 t}^{C}=\rho \\
& \widetilde{\zeta}_{t}^{C}=\zeta_{t} \\
& d_{0 t}^{C}=-\gamma_{t} \kappa_{1 t} \\
& d_{1 t}^{C}=-\gamma_{t} \kappa_{2 t} \\
& d_{2 t}^{C}=\gamma_{t} \kappa_{3 t} \\
& d_{3 t}^{C}=\gamma_{t} \varphi_{1 t}
\end{aligned}
$$

Cross-equation restrictions As before, given the forecasting rule (36), the $t-2$ conditional expectation of $(\mathrm{PC}-\mathrm{CF})$ is in the form

$$
\begin{aligned}
\mathbf{e}_{\pi}^{\prime} \mathbf{A}_{t-2}^{2} \widehat{\mathbf{z}}_{t-2}= & \widetilde{\rho}_{1, t-2}^{C} \mathbf{e}_{\pi}^{\prime} \mathbf{A}_{t-2} \widehat{\mathbf{z}}_{t-2}+(1-\tau) \widetilde{\rho}_{2, t-2}^{C} \mathbf{e}_{\pi}^{\prime} \widehat{\mathbf{z}}_{t-2}+\widetilde{\zeta}_{t-2}^{C} \mathbf{e}_{m c}^{\prime} \mathbf{K}_{t-2} \mathbf{A}_{t-2}^{2} \widehat{\mathbf{z}}_{t-2} \\
& +d_{0 t-2}^{C} \mathbf{e}_{\pi}^{\prime} \mathbf{K}_{t-2} \mathbf{A}_{t-2} \widehat{\mathbf{z}}_{t-2}+d_{1 t-2}^{C} \mathbf{e}_{\pi}^{\prime} \mathbf{K}_{t-2} \mathbf{A}_{t-2}^{2} \widehat{\mathbf{z}}_{t-2} \\
& +d_{2 t-2}^{C} \mathbf{e}_{\pi}^{\prime} \mathbf{K}_{t-2} \mathbf{A}_{t-2}^{3} \widehat{\mathbf{z}}_{t-2}+d_{2 t-2}^{C} \varphi_{1 t-2} \mathbf{e}_{\pi}^{\prime} \mathbf{K}_{t-2} \mathbf{J}_{t-2} \mathbf{A}_{t-2}^{4} \widehat{\mathbf{z}}_{t-2} \\
& +d_{3 t-2}^{C}\left(\mathbf{e}_{Q}^{\prime} \mathbf{K}_{t-2} \mathbf{J}_{t-2} \mathbf{A}_{t-2}^{2} \widehat{\mathbf{z}}_{t-2}+\mathbf{e}_{g^{y}}^{\prime} \mathbf{K}_{t-2} \mathbf{J}_{t-2} \mathbf{A}_{t-2}^{3} \widehat{\mathbf{z}}_{t-2}\right),
\end{aligned}
$$


where $\mathbf{K}_{t} \equiv\left(\mathbf{I}-\lambda_{t} \mathbf{A}_{t}\right)^{-1}$. Hence, the vector of cross-equation restrictions is given by

$$
\begin{aligned}
\mathbf{e}_{\pi}^{\prime} \mathbf{A}_{t-2}^{2}= & \tilde{\rho}_{1, t-2}^{C} \mathbf{e}_{\pi}^{\prime} \mathbf{A}_{t-2}+(1-\tau) \widetilde{\rho}_{2, t-2}^{C} \mathbf{e}_{\pi}^{\prime} \mathbf{I}+\widetilde{\zeta}_{t-2}^{C} \mathbf{e}_{m c}^{\prime} \mathbf{K}_{t-2} \mathbf{A}_{t-2}^{2} \\
& +d_{0 t-2}^{C} \mathbf{e}_{\pi}^{\prime} \mathbf{K}_{t-2} \mathbf{A}_{t-2}+d_{1 t-2}^{C} \mathbf{e}_{\pi}^{\prime} \mathbf{K}_{t-2} \mathbf{A}_{t-2}^{2} \\
& +d_{2 t-2}^{C} \mathbf{e}_{\pi}^{\prime} \mathbf{K}_{t-2} \mathbf{A}_{t-2}^{3}+d_{2 t-2}^{C} \varphi_{1 t-2} \mathbf{e}_{\pi}^{\prime} \mathbf{K}_{t-2} \mathbf{J}_{t-2} \mathbf{A}_{t-2}^{4} \\
& +d_{3 t-2}^{C}\left(\mathbf{e}_{Q}^{\prime} \mathbf{K}_{t-2} \mathbf{J}_{t-2} \mathbf{A}_{t-2}^{2}+\mathbf{e}_{g^{y}}^{\prime} \mathbf{K}_{t-2} \mathbf{J}_{t-2} \mathbf{A}_{t-2}^{3}\right) \\
\equiv & \mathbf{g}^{C}\left(\boldsymbol{\mu}_{t-2}, \mathbf{A}_{t-2}, \boldsymbol{\psi}\right) .
\end{aligned}
$$

\section{Appendix C: Estimates under non-time-varying coefficients}

Table C.1 below displays the NKPC's deep structural parameter estimates when we remove the time variation in the NKPC coefficients - and hence, these coefficients do not depend on time-varying inflation trend - and terms involving higher order leads of inflation. The resulting NKPC in DE specification is now as in (29), with the CF specification given by

$$
\widehat{\pi}_{t}=\rho \tau \widehat{\pi}_{t-1}+\rho(1-\tau) \widehat{\pi}_{t-2}+\zeta E_{t} \sum_{j=0}^{\infty} \beta^{j} \widehat{m c}_{t+j},
$$

where $\zeta=(1-\alpha)(1-\alpha \beta) /(\alpha+\alpha \theta \omega)$. The matrix $\mathbf{A}_{t}$, however, is still assumed to be time-varying and the two corresponding sets of restrictions in $\mathcal{F}_{t}^{j}$, for $j \epsilon\{D, C\}$, are still imposed.

Table C.1: Structural parameter estimates (median and 90\% confidence interval)

Sample period: 1960.Q1-2003.Q4

(constant coefficients and removing higher-order leads)

\begin{tabular}{|l|c|c|c|c|}
\hline & $\rho$ & $\alpha$ & $\theta$ & $\tau$ \\
\hline \hline DE_const & 0 & 0.561 & 12.17 & 1 \\
& $(0,0.11)$ & $(0.43,0.66)$ & $(7.96,15.31)$ & - \\
\hline DE_unconst & 0.69 & 0.613 & 11.75 & 0.58 \\
& $(0.36,1)$ & $(0.52,0.73)$ & $(10.23,13.96)$ & $(0.31,0.81)$ \\
\hline CF_const & 0.40 & 0.771 & 11.00 & 1 \\
& $(0.16,0.66)$ & $(0.67,0.84)$ & $(8.69,15.50)$ & - \\
\hline CF_unconst & 0.87 & 0.863 & 12.33 & 0.68 \\
& $(0.72,0.99)$ & $(0.76,0.91)$ & $(10.80,18.24)$ & $(0.480 .90)$ \\
\hline
\end{tabular}

Notes: (1) numbers in parentheses are $90 \%$ confidence intervals; (2) DE_const and $C F_{-}$const correspond to difference equation (DE) and closed-form (CF) specifications with $\tau=1$; (3) DE unconst and $C F_{-}$unconst correspond to $\mathrm{DE}$ and $\mathrm{CF}$ cases with unconstrained $\tau$, respectively; (4) DE_const is the benchmark case in Cogley and Sbordone (2008). 


\section{References}

[1] Benigno, P. and J.D. López-Salido, 2006. "Inflation persistence and optimal monetary policy in the Euro area," Journal of Money, Credit and Banking 38(3), pp. 587-614, April.

[2] Calvo, G., 1983. "Staggered prices in a utility-maximizing framework," Journal of Monetary Economics 12(3), pp. 383-398, September.

[3] Christiano, L., M. Eichenbaum, and C. Evans, 2005. "Nominal rigidities and the dynamic effects of a shock to monetary policy", Journal of Political Economy 113, pp. 1-45.

[4] Cogley, T. and T.J. Sargent, 2005. "Drifts and volatilities: Monetary policies and outcomes in the post WWII U.S." Review of Economic Dynamics 8(2), pp. 262-302.

[5] Cogley, T. and T.J. Sargent, 2008. "Anticipated utility and rational expectations as approximations of Bayesian decision making," International Economic Review 49(1), pp. 185-221, February.

[6] Cogley, T. and A.M. Sbordone, 2008. "Trend inflation, indexation, and inflation persistence in the New Keynesian Phillips Curve," American Economic Review 98(5), pp. 2101-2126.

[7] Fuhrer, J., 2009. "Inflation persistence," Federal Reserve Bank of Boston Working Paper.

[8] Fuhrer, J. and G. Moore, 1995. "Inflation persistence," The Quarterly Journal of Economics 110(1), pp. 127-59, February.

[9] Fuhrer, J., G. Moore, S. Schuh, 1995. "Estimating the linear-quadratic inventory model Maximum likelihood versus generalized method of moments," Journal of Monetary Economics 35(1), pp. 115-157, February.

[10] Fuhrer, J. and G. Olivei, 2005. "Estimating forward-looking Euler equations with GMM estimators: an optimal-instruments approach," Proceedings, Board of Governors of the Federal Reserve System (U.S.), pp. 87-114.

[11] Galí, J. and M. Gertler, 1999. "Inflation dynamics: A structural econometric analysis," Journal of Monetary Economics 44(2), pp. 195-222.

[12] Galí, J., M. Gertler, and J.D. López-Salido, 2005. "Robustness of the Estimates of the Hybrid New Keynesian Phillips Curve" Journal of Monetary Economics 52, pp. 1107-118. 
[13] Gouriéroux, C. and A. Monfort, 1995. Statistics and Econometric Models, Vol. 1. Cambridge University Press, Cambridge.

[14] Gouriéroux, C., A. Monfort, and A. Trognon, 1985. "Moindres carrés asymptotiques," Annales de l'inséé 58, pp. 91-122.

[15] Hayashi, F., 2000. Econometrics, Princeton University Press.

[16] Ireland, P. N., 2007. "Changes in the Federal Reserve's inflation target: Causes and consequences," Journal of Money, Credit and Banking 39(8), pp. 1851-1882, December.

[17] Kodde, A.D., F.C. Palm, and G.A. Pfann, 1990. "Asymptotic least-squares estimation efficiency considerations and applications," Journal of Applied Econometrics 5(4), pp. 229-243.

[18] Kozicki, S. and P. Tinsley, 2002. "Alternative sources of the lag dynamics of inflation," in Price Adjustment and Monetary Policy, pp. 3-47. Bank of Canada Conference Proceedings.

[19] Kreps, D., 1998. "Anticipated utility and dynamic choice," 1997 Schwartz Lecture, in Frontiers of Research in Economic Theory, D.P. Jacobs, E. Kalai, and M. Kamien (eds.), Cambridge University Press.

[20] Rudd, J. and K. Whelan, 2006. "Can rational expectations sticky-price models explain inflation dynamics?" American Economic Review 96(1), pp. 303-320, March.

[21] Sbordone, A.M., 2002. "Prices and unit labor costs: a new test of price stickiness," Journal of Monetary Economics 49(2), pp. 265-292, March

[22] Steinsson, J., 2003. "Optimal monetary policy in an economy with inflation persistence," Journal of Monetary Economics 50(7), pp. 1425-1456, October.

[23] Woodford, M., 2003. Interest and Prices, Princeton University Press. 